\title{
Luminous efficiency of meteors derived from ablation model after assessment of its range of validity
}

\author{
E. Drolshagen ${ }^{1 *}$, T. Ott ${ }^{1 *}$, D. Koschny ${ }^{2,3}$, G. Drolshagen ${ }^{1}$, J. Vaubaillon ${ }^{4}$, F. Colas ${ }^{4}$, B. Zanda ${ }^{5,4,6}$, S. Bouley ${ }^{7,4,6}$, S

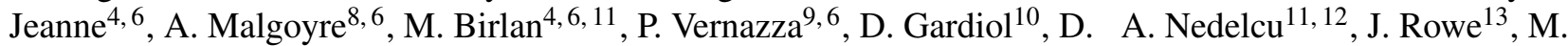 \\ Forcier $^{14,15}$, J. M. Trigo-Rodriguez ${ }^{16,17,18}$, E. Peña-Asensio ${ }^{17}$, H. Lamy ${ }^{19,20}$, L. Ferrière ${ }^{21,22}$, D. Barghini ${ }^{10,23}$, A. \\ Carbognani $^{24}$, M. Di Martino ${ }^{10}$. Rasetti ${ }^{10}$, G. B. Valsecchi ${ }^{25}{ }^{26}$, C. A. Volpicelli ${ }^{10}$, M. Di Carlo ${ }^{10}$, C. Knapic ${ }^{10}$, G. \\ Pratesi $^{10,28}$, W. Riva ${ }^{33}$, G. M. Stirpe ${ }^{10}$, S. Zorba ${ }^{10}$, O. Hernandez ${ }^{14,15}$, A. Grandchamps ${ }^{14,15}$, E. Jehin ${ }^{19,27}$, M. \\ Jobin $^{14,15}$, A. King ${ }^{13,29}$, A. Sanchez-Lavega ${ }^{30,31}$, A. Toni ${ }^{32,2}$, A. Rimola ${ }^{34}$, and B. Poppe ${ }^{1}$
}

(Affiliations can be found after the references)

Received March 22, 2021

\section{ABSTRACT}

Context. The luminous efficiency $\tau$ can be used to compute the pre-atmospheric masses of meteoroids from corresponding recorded meteor brightnesses. The derivation of the luminous efficiency is non-trivial and subject to biases and model assumptions. This has led to greatly varying results in the last decades of studies.

Aims. The present paper aims to investigate how a reduction of various observational biases can be achieved to derive (more) reliable values for the luminous efficiency.

Methods. 281 meteors observed by the FRIPON (Fireball Recovery and InterPlanetary Observation Network) are studied. The luminous efficiencies of the events are computed using an ablation-based model. The relations of $\tau$ as a function of the pre-atmospheric meteoroid velocity, $v_{e}$, and mass, $M_{e}$, are studied. Various aspects which could render the method less valid or cause inaccuracies or bias the results are investigated. On this basis the best suitable meteors were selected for luminous efficiency computations.

Results. The presented analysis shows the limits of the used method. The most influential characteristics that are necessary for reliable results for the $\tau$ computation were identified. The method is only valid for events observed in the continuum flow region of the atmosphere, with a low final height $h_{\text {final }}$, and a sufficiently high deceleration. For the consolidated dataset, $\tau$ is derived as a function of $M_{e}$ and $v_{e}$. We study the dependency of $\tau$ on the assumed meteoroid's density $\rho$ and include improved $\rho$-values for objects with identified meteoroid stream association. Based on the discovered individual biases and constrains we create a pre-debiased subset of 54 well-recorded events with a relative velocity change $>80 \%, h_{\text {final }}<70 \mathrm{~km}$, and a Knudsen number $\mathrm{Kn}<0.01$. We find $\tau$-values in the range between $0.012 \%$ and $1.1 \%$ for this pre-debiased subset and relations of $\tau$ and $M_{e}$ and $v_{e}$ of: $\tau=7.33 \cdot v_{e}^{-1.10}$ and $\tau=0.28 \cdot M_{e}^{-0.33}$. The $\tau-M_{e}$ relation is in good agreement with recent studies. The $\tau-v_{e}$ relation can be explained by the rather low velocity of the individual fireballs of the subset. These events radiate less efficiently since the second component of higher temperature only occurs with fast objects.

Conclusions. The derived luminous efficiency of meteoroids depends on the assumed material density. Our results indicate that the applied debiasing method improves the analysis of $\tau$ from decelerated meteoroids. The underlying method is only valid for meteors in the continuum-flow regime. These events tend to have low end heights, large masses, and high deceleration.

Key words. meteorites - meteors - meteoroids - minor planets - asteroids - comets - Techniques: photometric - atmospheric effects - data analysis

\section{Introduction}

Pre-atmospheric meteoroid mass computations are one of the most commonly investigated parameters of meteor research. To determine this mass, one possible technique is based on optical observation of the main meteor features: the position and the brightness. Traditionally, the mass of the corresponding meteoroid is then inferred using the luminous efficiency $\tau$. It describes the fraction of the object's loss of kinetic-energy that is converted into its brightness.

To this day the determination of $\tau$ depends on assumptions for unknown, hard to obtain parameters like the assumed density or shape of the entering object. Since even small variations in the assumed value for $\tau$ affect the computed meteoroid's mass to a high degree as shown e.g. by Koschny et al. (2017) precise values of the luminous efficiency are a vital factor in meteor research. Various approaches have been proposed to determine the luminous efficiency, see e.g. Verniani (1965), Ceplecha \& McCrosky (1976), Halliday et al. (1996), Hill et al. (2005), Weryk \& Brown (2013), Koschny et al. (2017), or Subasinghe et al. (2017). The main limitations of these studies tend to be a rather small dataset and $\tau$-values that vary by orders of magnitude between the studies.

Several studies have been published on the relationship between the luminous efficiency and the velocity of the meteoroid. Only a few deal with other meteoroid parameters. A more recent study by Drolshagen et al. (2021, accepted) provides an overview of the dependencies on e.g. the meteoroid's preatmospheric mass, velocity, entry angle, and composition.

Reinforcing the significance of work done e.g. by Subasinghe \& Campbell-Brown (2018) and Čapek et al. (2019) this work focuses on the dependency of $\tau$ and the 
pre-atmospheric meteoroid mass. Subasinghe \& CampbellBrown (2018) applied the numerical approach of Subasinghe et al. (2017) to 12 non-fragmenting meteors recorded by the Canadian automated meteor observatory (CAMS, see e.g. Weryk \& Brown (2013)). The masses of the small non-iron meteoroids were on the order of $10^{-6} \mathrm{~kg}-10^{-4} \mathrm{~kg}$ and they found luminous efficiencies in the range of $10^{-2} \%-10^{2}$ $\%$. Moreover, they found a weak relationship between $\tau$ and the initial meteoroid mass. It should be mentioned that their data showed large scattering and only 12 meteors were taken into consideration with all limitations of such a small dataset (Subasinghe \& Campbell-Brown 2018). Čapek et al. (2019) found similar results for faint, slow, and low altitude meteors. They report on the observation and modelling of the light curves of 53 meteors that were recorded with double station video observations and associated to small iron meteoroids.

Drolshagen et al. (2021, accepted) utilized the data of the originally French Fireball Recovery and InterPlanetary Observation Network (FRIPON) to derive the luminous efficiencies of the observed meteors' corresponding meteoroids. The study focused on a subset of 294 fireballs (data from 2016 - 2020). The results still show considerable uncertainty with regards to the derived values for the luminous efficiencies. They range from $10^{-4} \%$ to $100 \%$, whereas about $70 \%$ of them are in the order of $0.1 \%-10 \%$.

Drolshagen et al. (2021, accepted) reported a dependence of $\tau$ on the initial velocity $v_{e}$ of the meteor given by the relation $\tau=0.0023 \cdot v_{e}^{2.3}$. Furthermore, as in previous studies, they found that smaller meteoroids radiate more efficient: $\tau=0.48 \cdot M_{e}^{-0.47}$, with the pre-atmospheric meteoroid's mass $M_{e}$. They also obtained a weak link between the luminous efficiency and the angle of entry $\gamma: \tau=0.80 \cdot 10^{-0.0075 \cdot \gamma}$.

This paper takes a new look at different aspects of possible uncertainties and their influences on the derivation of the luminous efficiency. One of those, noted in Drolshagen et al. (2021, accepted), is the bulk density of meteoroids assumed for the calculation of the pre-atmospheric masses. The influence of the density value on the results were studied by using different assumptions for the complete dataset as well as for individual exemplary events. The density can be refined by considering additional meteoroid parameters such as links to meteoroid streams. If the parent body is known, the density of the associated meteoroids is often available. This leads to more accurate stream meteoroid bulk densities and pre-atmospheric masses. Many studies have been published on ways to identify possible shower associations of detected meteors. Southworth \& Hawkins (1963) introduced the $D$-criterion which, in spite of its age, is still widely applied and computationally practical. This criterion is used to compute the similarity between the orbits of two meteoroids by measuring the distance between these orbits with a similarity function. In the dataset of 281 meteors analyzed in this study 99 were found to have a shower association based on the $D$-criterion. Utilizing the densities found this way within the mass computation and combining it with the method presented in Gritsevich \& Koschny (2011) to derive $\tau$ should provide improved results.

Moreover, this paper examines an observational bias due to missing parts of the trajectory. Especially for faint or very fast meteors it is possible that only part of the trajectory was captured by the cameras. The effect of incomplete light curves was investigated with simulated meteors. It was found that the method presented herein is surprisingly stable with respect to missing data points.

Another important limitation to be considered is the applied method itself which is based on the work by Gritsevich (2008). It is based on parameters that are linked to physical properties of the entering object, including amongst others, its shape, composition, mass, velocity, ablation efficiency, or even to the corresponding meteor's duration. The main assumption of the model is that ablation of the entering meteoroid is the main interaction process with the atmosphere. Fragmentation is neglected in this approach which is certainly a constraint for its applicability. This study aims to investigate the range of validity of the method.

Various previous studies build on the method by Gritsevich (2008). Gritsevich \& Koschny (2011) utilized the approach and presented a method to determine the luminous efficiency $\tau$ of recorded meteors with a model fitted to the observed light curve. In line with Drolshagen et al. (2021, accepted) this method is used in this work as well.

One parameter studied in more detail in this work is the final height of the recorded objects. As mentioned in Drolshagen et al. (2021, accepted), it is expected that especially objects with low end heights undergo significant deceleration during the observation period. Since the used method of Gritsevich (2008) is based on the measured deceleration of the events, it might not be valid for all meteors; especially objects which show high end heights tend to be less decelerated. In addition, it is possible that the lack of deceleration in the data points towards instrumentation limitations manifested in positional imprecisions. With this in mind, the dependency of the final height and the computed luminous efficiency is investigated and discussed. To analyse possible observational biases, a subset of the data is created using only events for which we expect that they can be well recorded by FRIPON and analysed using the Gritsevich (2008) method. This subset consists of qualitatively good data making these events the most reliable. As expected, small objects tend to exhibit larger $\tau$-values and higher end heights. Taking only objects into account which penetrated deep into the atmosphere excludes most events with small pre-atmospheric masses and apparently large luminous efficiencies. This test revealed that the negative linear correlation in $\log -\log$ space of $\tau$ and the pre-atmospheric meteoroid's mass is quite similar for this subset.

We demonstrate that there is a similar dependency between the relative velocity change and the luminous efficiency by investigating the influence of deceleration on the results using the same strategy.

An important gas characteristic is the mean free path length of its molecules which is used e.g. to identify certain flow regimes to describe the interaction with objects passing through it. It can be used to describe the Earth's atmosphere: At very high altitudes there are almost no collisions between the air molecules (free flow). With decreasing altitude, the number of collisions between the molecules increases until the surrounding gas can be considered as a continuous medium.

As described by e.g. Bronshten (1983), Moreno-Ibáñez et al. (2018), or Popova (2000), a meteoroid passes different flow 
regimes during its way through the atmosphere. Since the number of collisions with the surrounding air molecule affects the meteoroid it relates to the meteor phenomenon.

The flow regimes are applicable to a gas when it is studied within a characteristic length, in this case to a body immersed in the atmosphere. When the mean free path is small in comparison with the dimension of the flow field or the dimension of the body in the flow field, then the gas can be considered as a continuum and the ordinary gas dynamics (continuum mechanics) is applicable for the analysis of the gas flow. If the mean free path is large compared to the dimension of the body, then the effects of the discrete character of the gas must be taken into account and a statistical approach is needed.

It is expected that the underlying theory used by Gritsevich (2008) is based on ordinary gas dynamics and only valid for events observed in the continuum flow regime. In this regard, it is clear that these equations cannot model the mechanical behaviour of a body immersed in a gas when the mean free path is not small enough.

The events observed with FRIPON do appear at different heights and have different sizes and velocities. Hence, their interaction with the atmosphere is different. One approach to solve this problem is to separate the dataset by flow regimes. The flight of the meteoroid in the atmosphere could experience four flow regimes: The free molecular regime, the transitional regime, the slip-flow regime, and the continuum-flow regime (Moreno-Ibáñez et al. 2018). They are usually distinguished based on the Knudsen number, defined by the ratio of the mean free path length of the molecules and a characteristic size of the meteoroid (in most cases its radius). Most of the events in our dataset lie within the transitional flow regime and 93 in the continuum-flow regime.

This study documents a comprehensive analysis of all of the before mentioned aspects. Importantly, our findings made it possible to select a pre-debiased subset for which the derived luminous efficiency values can be expected to be of good quality. This subset includes only meteors that occur in the continuum-flow regime and tend to have low end heights, large masses, and a large deceleration during the observed trajectory.

In Section 2 a brief overview is given on the utilized data and applied methods. The density influence on the luminous efficiency is discussed in Section 3 and the results when taking the stream association into account are presented in Section 4. In Section 5 the possibility of an observational bias due to missing parts of the trajectory is investigated. Section 6 examines the influence of the derived luminous efficiency on the objects' final heights and Section 7 on the deceleration. We discuss the different flow regimes in Section 8. Section 9 presents a discussion and is followed by a short conclusion in Section 10 .

\section{Data and Methods}

In Drolshagen et al. (2021, accepted) a method to derive the luminous efficiency $\tau$ and the shape change coefficient $\mu$ of meteors recorded by FRIPON was presented. The method is based on the work by Gritsevich \& Koschny (2011). The final dataset examined by Drolshagen et al. (2021, accepted) includes 294 meteors. The data and data processing to derive the parameters are explained in detail in Drolshagen et al. (2021, accepted). In the following subsections a short summary is presented introducing the data and the adaptation of the method used in this work .

\subsection{The FRIPON data}

The originally French network FRIPON (Fireball Recovery and InterPlanetary Observation Network) is designed for the detection of fireballs and meteorite recovery. It consists of all-sky cameras covering the sky over France as well as parts of the neighbouring countries. The stations have a distance between each other of roughly $80 \mathrm{~km}$ and a fireball that was detected by at least two stations simultaneously is stored and processed automatically. The cameras are operated with 30 frames per second during the nights. Every 10 minutes one 5 seconds long exposure is taken for calibration purposes. The network is introduced in Colas et al. (2014) and first results and statistics were recently published in Colas et al. (2020). In Audureau et al. (2014) the used camera control and meteor event detection software, FreeTure, is presented.

To compute the pre-atmospheric mass of the recorded meteoroids and asteroids the FRIPON pipeline utilizes an optimization of a fit through the velocity data, which is a slightly modified method of the one introduced by Gritsevich (2008). For details about the data processing we refer to Jeanne et al. (2019).

The same set of FRIPON meteors selected in Drolshagen et al. (2021, accepted) is utilized in this work. They were collected between April 2016 and June 2020. For this time range the FRIPON database consists of 3871 confirmed events. In the previous work a small subset of 294 was chosen based on sufficient event data, physically realistic results, and good quality light curve fits. For the included meteoroids masses in the range of $10^{-6} \mathrm{~kg}-100 \mathrm{~kg}$ luminous efficiency values in the range of $10^{-4} \%-100 \%$ were computed. Due to the different starting conditions for the fit to the light curve, this work is based on a slightly different subsets of 281 events, for which details are reported in Section 4.

As explained in Drolshagen et al. (2021, accepted), due to different assumptions, the pre-atmospheric mass values presented herein differ from the ones determined by the FRIPON pipeline. One of this assumptions is e.g. the shape change coefficient for which a value of $0 . \overline{6}$ is assumed within the FRIPON pipeline. For the same density assumption, our values are about half an order of magnitude larger than theirs. This highlights how strongly the density assumption influences the results. The difference between the masses computed by the FRIPON pipeline and within this study is shown using a histogram in Figure A.1 in the Appendix.

\subsection{Computation of the luminous efficiency and the shape change coefficient}

Our method to compute the mass of an entering meteoroid is a variation on the procedure introduced by Gritsevich (2008). The origin of the method is based on the work by Stulov et al. (1995), and the adapted variations to the methodology are detailed in Jeanne et al. (2019) and Drolshagen et al. (2021, accepted). The mass is computed based on the measured velocity and height of the recorded meteor. To do so, two dimensionless parameters were calculated: the ballistic coefficient $\alpha$ and the mass loss parameter $\beta$. 
$\alpha=\frac{c_{d} \cdot \rho_{0} \cdot h_{0} \cdot S_{e}}{2 \cdot M_{e} \cdot \sin (\gamma)}$
$\beta=\frac{(1-\mu) \cdot c_{h} \cdot v_{e}^{2}}{2 \cdot c_{d} \cdot H}$

with the parameters the meteoroid had prior to its entry into the atmosphere: mass $M_{e}$, velocity $v_{e}$, and cross section area $S_{e}$. Furthermore, the angle between horizon and trajectory, $\gamma$, is taken into account as well as some physical constants: the drag coefficient $c_{d}$, the heat-transfer coefficient $c_{h}$, the effective destruction enthalpy $H$, the gas density at sea level $\rho_{0}$ (presumed to be $1.29 \mathrm{~kg} / \mathrm{m}^{3}$ ), and the scale height $h_{0}$ (presumed to be $7160 \mathrm{~m}$ ). Moreover, $\beta$ depends on the shape change coefficient $\mu$.

With these parameters $M_{e}$ can be obtained with Eq. (3),

$$
M_{e}=\left(\frac{c_{d} \cdot A_{e} \cdot \rho_{0} \cdot h_{0}}{2 \cdot \alpha \cdot \sin (\gamma) \cdot \rho^{2 / 3}}\right)^{3},
$$

including the pre-atmospheric shape factor of the meteoroid $A_{e}$ and the meteoroid bulk density $\rho$. At this point, some assumptions have to be made for $A_{e}, c_{d}$, and $\rho$. Gritsevich \& Koschny (2011) supposed $c_{d}=1.2$ and $A_{e}=1.5$, which will be assumed in this work, too. A density value of $\rho=2500 \mathrm{~kg} / \mathrm{m}^{3}$ was defined for impact risk assessments of satellites by the European Cooperation for Space Standardization (ECSS) (ECSS 2008). This value was used in Drolshagen et al. (2021, accepted). In this study it will only be used for comparisons.

In the next step the method introduced by Gritsevich \& Koschny (2011) was used to derive the luminous efficiencies $\tau$ and shape change coefficients $\mu$ of the recorded objects. This was done by applying a least-square fit of Eq. (4) to the observed light curves $I\left(v^{*}\right)$.

$$
I\left(v^{*}\right)=\frac{\tau \cdot M_{e} \cdot v_{e}^{3} \cdot \sin (\gamma) \cdot f\left(v^{*}\right)}{2 \cdot h_{0}}
$$

with

$f\left(v^{*}\right)=v^{* 3} \cdot\left(\overline{E i}(\beta)-\overline{E i}\left(\beta \cdot v^{* 2}\right)\right) \cdot\left(\frac{\beta \cdot v^{* 2}}{1-\mu}+1\right) \cdot \exp \left(\frac{\beta \cdot\left(\mu \cdot v^{* 2}-1\right)}{1-\mu}\right)$

the exponential integral $\overline{E i}(x)$ :

$\overline{E i}(x)=\int_{\infty}^{x} \frac{e^{z}}{z} d z$

and the dimensionless velocity:

$v^{*}=\frac{v}{v_{e}}$.

We mark dimensionless parameters with an asterisk. They were divided by the corresponding pre-atmospheric values and in consequence range between zero and one, see Eq. (7) for the dimensionless velocity $v^{*}$ as an example. To illustrate the method, four exemplary light curves of corner case characteristics with resulting fit are presented in Fig. 1.

\section{Densities and mass computation}

One of the main results of the FRIPON pipeline is the preatmospheric mass of a recorded fireball event. In the course of this work we investigated the influence of the assumed density on these masses. The range of values that can be assumed for the meteoroid bulk density is wide. Not only the assumed ECSS standard density but also specific object types' densities are uncertain.

For example, the densities of recovered meteorites already vary strongly. According to Macke (2010) they range from e.g. about $2500 \mathrm{~kg} / \mathrm{m}^{3}-3500 \mathrm{~kg} / \mathrm{m}^{3}$ for ordinary chondrites, for carbonaceous chondrites from about $1500 \mathrm{~kg} / \mathrm{m}^{3}-5000$ $\mathrm{kg} / \mathrm{m}^{3}$, for pallasites from about $2500 \mathrm{~kg} / \mathrm{m}^{3}-7500 \mathrm{~kg} / \mathrm{m}^{3}$, for mesosiderites from $3000 \mathrm{~kg} / \mathrm{m}^{3}-7000 \mathrm{~kg} / \mathrm{m}^{3}$ and from 4000 $\mathrm{kg} / \mathrm{m}^{3}-8000 \mathrm{~kg} / \mathrm{m}^{3}$ for iron meteorites. However, it has to be kept in mind that the more porous (parts of) extra-terrestrial objects do not survive their way through the atmosphere as unscathed as more solid objects or parts.

For some asteroids in our solar system the densities are well established. Following Milone \& Wilson (2014), Ceres has a density of about $2090 \mathrm{~kg} / \mathrm{m}^{3}$, Pallas one of ca. $2570 \mathrm{~kg} / \mathrm{m}^{3}$, Juno of around $3200 \mathrm{~kg} / \mathrm{m}^{3}$, Vesta of almost $3500 \mathrm{~kg} / \mathrm{m}^{3}$, and Eros of roughly $2670 \mathrm{~kg} / \mathrm{m}^{3}$. For comets, densities are even lower, as investigated e.g. by Pätzold et al. (2016) for the Jupiter-family comet $67 \mathrm{P} /$ Churyumov-Gerasimenko. They showed that the nucleus of the comet is a dusty body, with a high porosity of ca. $73 \%$ and a low bulk density of about $533 \mathrm{~kg} / \mathrm{m}^{3}$. In Gritsevich \& Koschny (2011) the meteoroid density was estimated to be between $1000 \mathrm{~kg} / \mathrm{m}^{3}-4000 \mathrm{~kg} / \mathrm{m}^{3}$ if no further information about the object is available. One common assumption is $3500 \mathrm{~kg} / \mathrm{m}^{3}$ (Levin 1956). Moorhead et al. (2017) presented a two-population distribution of the bulk density of sporadic meteoroids. They divided the analysed data on the basis of the Jupiter-based Tisserand parameter $T_{J}$ to classify the objects on the basis of their dynamic orbital origin (Levison \& Duncan (1994)), referring to Kikwaya et al. (2011) for the relationship between this parameter and the object's density. Kikwaya et al. (2011) determined the densities of recorded meteoroids by combining optical meteor observations with an ablation model and linking the results with the orbital parameters of the observed objects and possible parent bodies. Moorhead et al. (2017) applied a normal fit to both density distributions. They found a bulk density for objects with $T_{J}<2$, indicating that they originate from Halley-type comets, of $857 \mathrm{~kg} / \mathrm{m}^{3}$ and for meteoroids with $T_{J}>2$ a mean bulk density of $3792 \mathrm{~kg} / \mathrm{m}^{3}$.

Chondritic meteoroids exhibit different ablation behaviour than the most fragile cometary aggregates, and it is important to explain the origin of such differences in this context. Cometary aggregates exhibit low density and high porosity, just affecting their tensile strengths (Blum et al. 2006). Fireballs produced by $\mathrm{cm}$-sized meteoroids associated with cometary streams are often exhibiting disruptive flares with sudden increases in luminosity, or just an ending (castastrophic) flare. This behaviour, associated with the fragmentation of the meteoroid due to aerodynamic overpressure suffered during penetration into the denser atmospheric layers at hypervelocity, was used to infer the tensile strength of typical shower meteors (Trigo-Rodríguez \& Llorca 2006). There is a correlation that a low tensile strength (and low density) can be correlated with the degree of primitiveness of meteoroids (Trigo-Rodríguez \& Blum 2009). We expect that the 


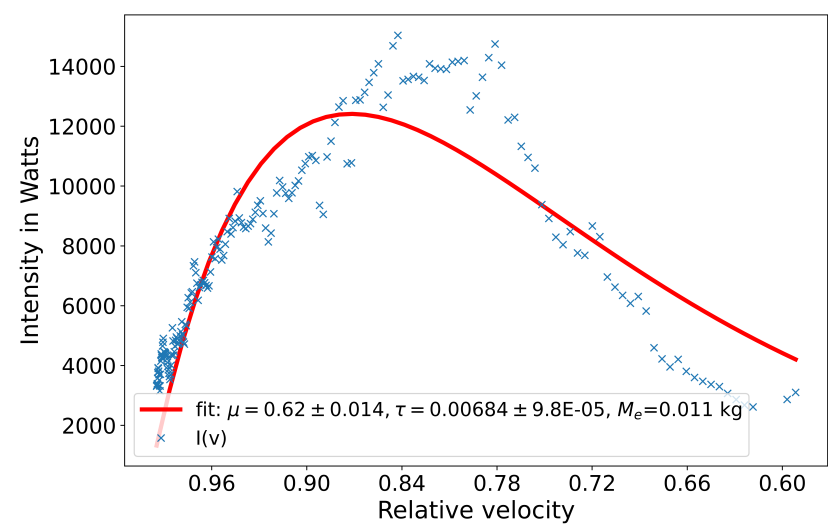

(a) Meteor from 1. December 2016

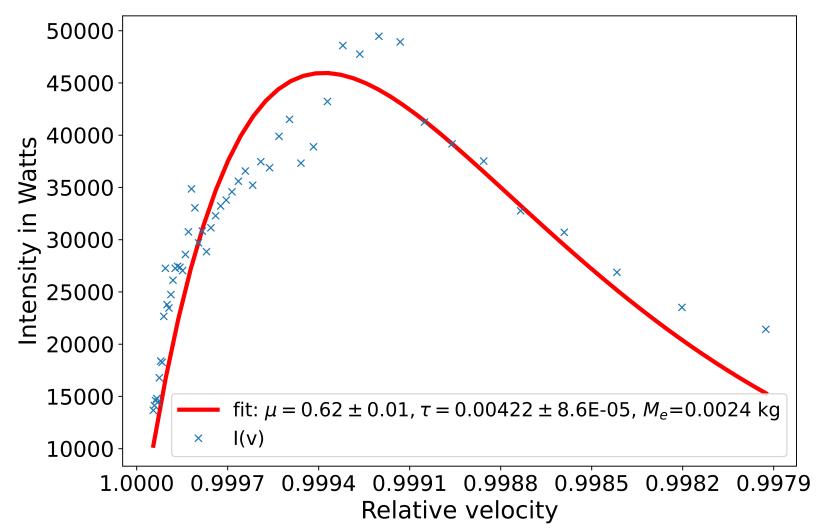

(c) Meteor from 18 August 2018

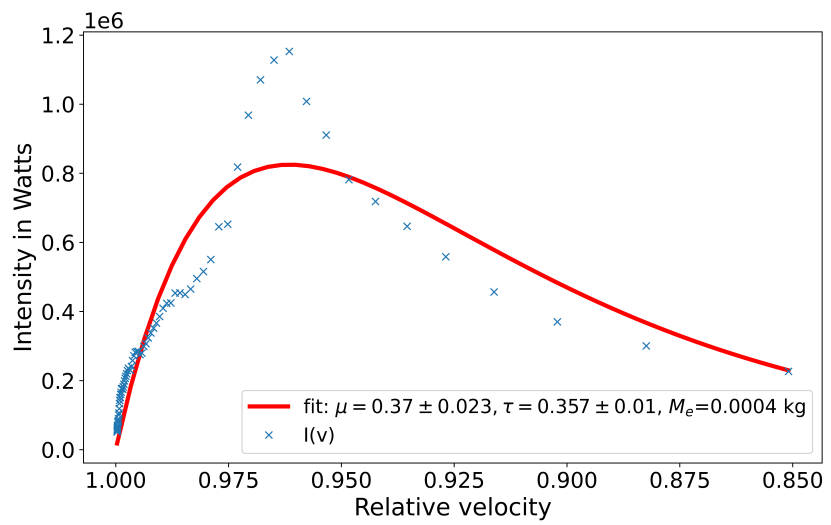

(b) Fireball from 21 September 2017

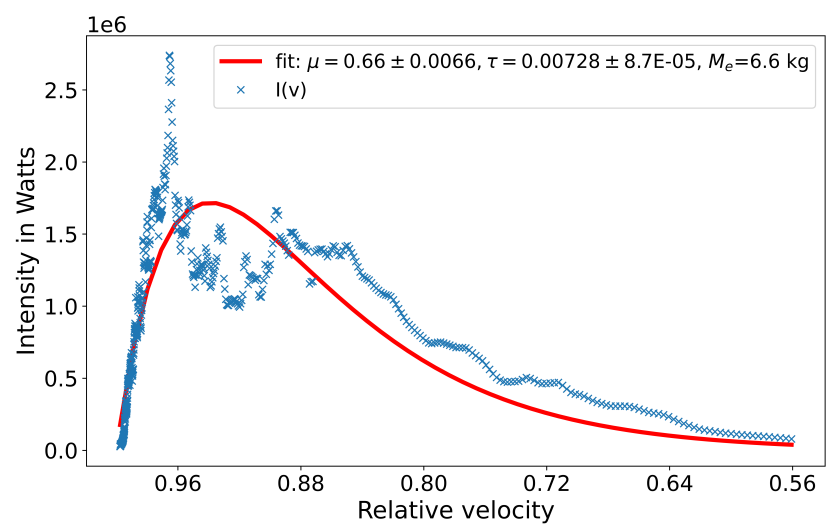

(d) Fireball from 16 October 2019

Fig. 1: Computed light curves of the four examplary FRIPON events presented in Table 1. with applied fit. The $\mathrm{x}$-axis displays the relative velocity of the event for which each velocity value is divided by the events's initial velocity. The brightness values are shown as blue ' $x$ '. The applied fit is displayed as solid red line. a): the meteor recorded on 1. December 2016 at 20:33:58 UT; b): the fireball from 21 September 2017 (22:58:28 UT); c): the meteor detected on 18 August 2018 at 22:47:29 UT; d): the fireball from 16 October 2019 at 18:57:30 UT.

luminous efficiency of these bolides is higher because: 1) The meteoroids are quickly disrupted into micron-sized grains that are released and affected by the thermal wave in the meteoroid head (Trigo-Rodríguez 2019), 2) Most of these meteoroid streams are of cometary origin so they exhibit higher inclination and eccentricity that result in higher geocentric velocity than the typical one for meteoroids produced by main belt asteroids. In consequence, the release of the meteoroid grains at higher velocity produce very bright flares due to the sudden ablation of the most volatile mineral phases, like e.g. Na (Trigo-Rodríguez \& Llorca 2007b).

As published in Colas et al. (2020) the FRIPON dataset contains many shower meteors, which can usually be traced back to comets. Hence, a lower density would probably be more suitable. They classified about $45 \%$ of the recorded objects as shower meteors. Following the ECSS (2008), a density of $\rho=$ $2500 \mathrm{~kg} / \mathrm{m}^{3}$ was assumed for the computations carried out in Drolshagen et al. (2021, accepted). In this paper we will rely on shower associations for most of the density estimations, for sporadic meteors a density of $1300 \mathrm{~kg} / \mathrm{m}^{3}$ in agreement with Narziev (2019) will be assumed.
For further investigations on the influence of the density on the results, the computations of all individual events of the complete dataset were repeated for a range of reasonable densities $\left(500 \mathrm{~kg} / \mathrm{m}^{3}-4500 \mathrm{~kg} / \mathrm{m}^{3}\right.$ (Blum et al. 2006)) and the differences noted. It turned out that a change of $\rho$ has little effect on $\mu$, but strongly influences $\tau$. The reason for this result is evident in Eq. (4) as $\tau$ and $M_{e}$ are both factors. Thus, a change of $\rho$ causes a change in pre-atmospheric mass. We found significant differences in the results of the density variations and believe that this emphasizes the influence and importance of the assumed density value for the derived luminous efficiency. Interestingly, we found a stronger correlation between the velocity and calculated $\tau$ for lower assumed densities for the density varied $\tau-v_{e}$ relations. E.g. for $\rho=500 \mathrm{~kg} / \mathrm{m}^{3}$ we found: $\tau=8.443 \cdot 10^{-5} \cdot v_{e}^{3.00}$ and for $\rho=4500 \mathrm{~kg} / \mathrm{m}^{3}: \tau=1.025 \cdot v_{e}^{0.80}$. The results of the $\tau-M_{e}$ relation vary without any obvious pattern. There are no trends in the slope parameters. For $\rho=500$ $\mathrm{kg} / \mathrm{m}^{3}$ we found: $\tau=0.232 \cdot M_{e}^{-0.54}$ and for $\rho=4500 \mathrm{~kg} / \mathrm{m}^{3}$ : $\tau=1.137 \cdot M_{e}^{-0.39}$. A table of the results of the density variations can be found in Table B.1 the Appendix and the corresponding plots in the supplementary material.

For a more structured study, four exemplary events were chosen from our dataset. All of them showed a light curve that 
matches the theoretical shape quite well. We chose one event for each of four categories: bright and slow, bright and fast, faint and slow, and faint and fast. The information of these events can be found in Table 1. The computed light curves with resulting fit are shown in Fig. 1.

The error estimations that are listed in Table 1 of the luminous efficiency and the shape change coefficient are statistical errors based on the fit as explained in Drolshagen et al. (2021, accepted). We also direct the reader to this paper for more details on the method to derive the peak brightness as well as its assumed error. For a discussion on the errors of the parameters that were generated by the FRIPON pipeline, we refer to Jeanne et al. (2019) and Colas et al. (2020).

We determined the luminous efficiencies and shape change coefficients for these four events, assuming various values of $\rho$ ranging from $1000 \mathrm{~kg} / \mathrm{m}^{3}$ up to $5000 \mathrm{~kg} / \mathrm{m}^{3}$. The results are presented in Fig. 2. As evident in Eq. (3), the mass is inversely proportional to the density by $M_{e} \propto \rho^{-2}$. Furthermore, the luminosity is proportional to the mass (Eq. (4)) and hence to the density by $I \propto M_{e} \propto \rho^{-2}$. If one converts the formula to $\tau$ it is also easy to see that $\tau \propto \rho^{2}$. This agrees with the curves shown in Fig. 2.

Furthermore, these dependencies explain the relatively small differences we see for the $\tau-M_{e}$ relations of different density assumptions. $\tau$ decreases with decreasing $\rho$, but $M_{e}$ increases with decreasing $\rho$, which at first might seem counter intuitive. The interaction of the proportionalities causes the data points, and consequently the fit through them, to shift towards higher masses and lower luminous efficiencies when the density is reduced and vice versa for increased densities.

This emphasizes the large effect of the luminous efficiency on the assumption of the density of the source object. Most of the well-studied meteoroid streams have known parent bodies. Incorporating this information into the mass computation delivers significantly better results, due to the more precise value for the density of a stream meteoroid. The inclusion of the shower affiliation of the recorded meteors, taking into account the known values of densities for the corresponding meteoroids of special events, is the next logical step for refining the results. This will be done in the following.

\section{Shower affiliation}

There exists a considerable body of literature on methods to determine the shower affiliation of recorded meteors. The most established one is the frequently used $D$-criterion as introduced by Southworth \& Hawkins (1963). It is based on a dissimilarity function of orbital parameters. Even though there are many variations based on this method, following Galligan (2001) the original is still functioning competently. A previous study of the FRIPON database applied an alternative approach, Colas et al. (2020) compared the observed radiant positions and velocities with those of established showers (based on the method of Micheli (2013) and Micheli et al. (2016)). For about $45 \%$ of the dataset they found a shower affiliation. Within this work the $D$ criterion as proposed by Southworth \& Hawkins (1963) will be used and briefly introduced in the following.

\subsection{D-criterion}

The basic principle of the $D$-criterion is to compute the similarity between two orbits of meteoroids. This is done by measuring the distance between these orbits with a similarity function - the $D$ criterion. To do so, most approaches utilize the orbital elements. Numerous studies investigated and adapted the $D$-criterion. They each apply the similarity function to different parameters. This results in different dimensions of the phase space in which the distance between the points (representing the meteoroids) is the distance between these orbits. Southworth \& Hawkins (1963) defined the $D_{S H^{-}}$-criterion for two orbits $A$ and $B$ as:

$$
\begin{aligned}
D_{S H}(A, B)^{2} & =\left(e_{B}-e_{A}\right)^{2}+\left(q_{B}-q_{A}\right)^{2}+\left(2 \cdot \sin \left(\frac{I_{A B}}{2}\right)\right)^{2} \\
& +\left(\frac{e_{A}+e_{B}}{2}\right)^{2} \cdot\left(2 \cdot \sin \left(\frac{\Pi_{A B}}{2}\right)\right)^{2}
\end{aligned}
$$

with

$$
\begin{aligned}
\left(2 \cdot \sin \left(\frac{I_{A B}}{2}\right)\right)^{2} & =\left(2 \cdot \sin \left(\frac{i_{B}-i_{A}}{2}\right)\right)^{2} \\
& +\sin \left(i_{A}\right) \cdot \sin \left(i_{B}\right)\left(2 \cdot \sin \left(\frac{\Omega_{B}-\Omega_{A}}{2}\right)\right)^{2}
\end{aligned}
$$

and

$\Pi_{A B}=\left(\Omega_{B}+\omega_{B}\right)-\left(\Omega_{A}+\omega_{A}\right)$

with the eccentricity $e$, the perihelion distance $q$, the inclination $i$, the argument of periapsis $\omega$, and the longitude of ascending node $\Omega$. The term $I_{A B}$ describes the difference between the two orbital planes and $\Pi_{A B}$ refers to the difference in the orbits' orientations within the planes.

Like $D_{S H}$ the similarity functions introduced by e.g. Drummond (1981) or Jopek (1993) measure the distances in a five dimensional phase. Depending on the number of parameters the dimension of the phase can vary. E.g. a seven-dimensional phase is utilized by Jopek et al. (2008), taking into account the differences between the orbital energies, the vectors of angular momentum, as well as the Laplace vectors. In an attempt to obtain a space with as many dimensions as there are physical parameters that can be measured independently from each other, Valsecchi (1999) introduced a distance function based on geocentrically observed parameters. Moreover, e.g. Rudawska (2015) introduced a new function based on geocentric parameters and added it to the $D_{S H}$-criterion. Many studies analysed and compared the different criteria. E.g. Jopek (1993) compared $D_{S H}$, as defined by Southworth \& Hawkins (1963), and the one of Drummond (1981) and found that $D_{S H}$ depends highly on the perihelion distance and the other on the eccentricity. Moreover, e.g. Moorhead (2016) analysed the performance of three different $D$-criteria, $D_{S H}$, the one of Drummond (1981), and Valsecchi (1999)'s criteria. She concluded that the criterion of Valsecchi (1999) might be the preferred one if one's focus is to exclude sporadics.

\subsection{Densities of Stream Meteoroids}

For various meteoroid streams the parent bodies have been identified and their densities studied. Since many meteor 
Table 1: Details about the four events presented in Fig. 1 and chosen as examples for a bright and slow, a bright and fast, a faint and slow, as well as for a faint and fast event, with the number of detecting stations, the computed meteor's pre-atmospheric velocity $v_{e}$, the pre-atmospheric meteoroid's mass $M_{e}$, the shape change coefficient $\alpha$, the mass loss parameter $\beta$, the angle between horizon and trajectory $\gamma$, as well as the determined maximum absolute magnitude, the derived shape change coefficient $\mu$, and the derived luminous efficiency $\tau$.

\begin{tabular}{|c|c|c|c|c|c|c|c|c|c|c|c|}
\hline Example & Date & $\begin{array}{l}\text { Time } \\
\text { in UT }\end{array}$ & $\begin{array}{l}\# \\
\text { Sta- } \\
\text { tions }\end{array}$ & $\begin{array}{l}v_{e} \text { in } \\
\mathrm{km} / \mathrm{s}\end{array}$ & $\begin{array}{l}M_{e} \text { in } \\
\mathrm{kg}\end{array}$ & $\alpha$ & $\beta$ & $\gamma$ in & $\begin{array}{l}\text { Peak } \\
\text { bright- } \\
\text { ness in } \\
\text { mag }\end{array}$ & $\mu$ & $\tau$ in $\%$ \\
\hline $\begin{array}{l}\text { Faint- } \\
\text { slow }\end{array}$ & $\begin{array}{l}2016- \\
12-01\end{array}$ & $20: 33: 58$ & 3 & 20.4 & 0.01 & 236.71 & 0.91 & 58.7 & $\begin{array}{l}-2.5 \\
\pm 0.5\end{array}$ & $\begin{array}{l}0.62 \\
\pm 0.014\end{array}$ & $\begin{array}{l}0.68 \\
\pm 0.0098\end{array}$ \\
\hline $\begin{array}{l}\text { Bright- } \\
\text { fast }\end{array}$ & $\begin{array}{l}2017- \\
09-21\end{array}$ & $22: 58: 28$ & 10 & 64.2 & 0.0004 & 970.02 & 10.75 & 39.1 & $\begin{array}{l}-7.2 \\
\pm 0.5\end{array}$ & $\begin{array}{l}0.37 \\
\pm 0.023\end{array}$ & $\begin{array}{l}36.65 \\
\pm 1\end{array}$ \\
\hline $\begin{array}{l}\text { Faint- } \\
\text { fast }\end{array}$ & $\begin{array}{l}2018- \\
08-18\end{array}$ & $22: 47: 29$ & 4 & 65.2 & 0.002 & 658.56 & 383.77 & 30.7 & $\begin{array}{l}-3.8 \\
\pm 0.5\end{array}$ & $\begin{array}{l}0.62 \\
\pm 0.01\end{array}$ & $\begin{array}{l}0.42 \\
\pm 0.0086\end{array}$ \\
\hline $\begin{array}{l}\text { Bright- } \\
\text { slow }\end{array}$ & $\begin{array}{l}2019- \\
10-16\end{array}$ & $18: 57: 30$ & 4 & 14.9 & 6.6 & 58.64 & 2.61 & 24.2 & $\begin{array}{l}-8.1 \\
\pm 0.5\end{array}$ & $\begin{array}{l}0.66 \\
\pm 0.0066\end{array}$ & $\begin{array}{l}0.73 \\
\pm 0.0087\end{array}$ \\
\hline
\end{tabular}

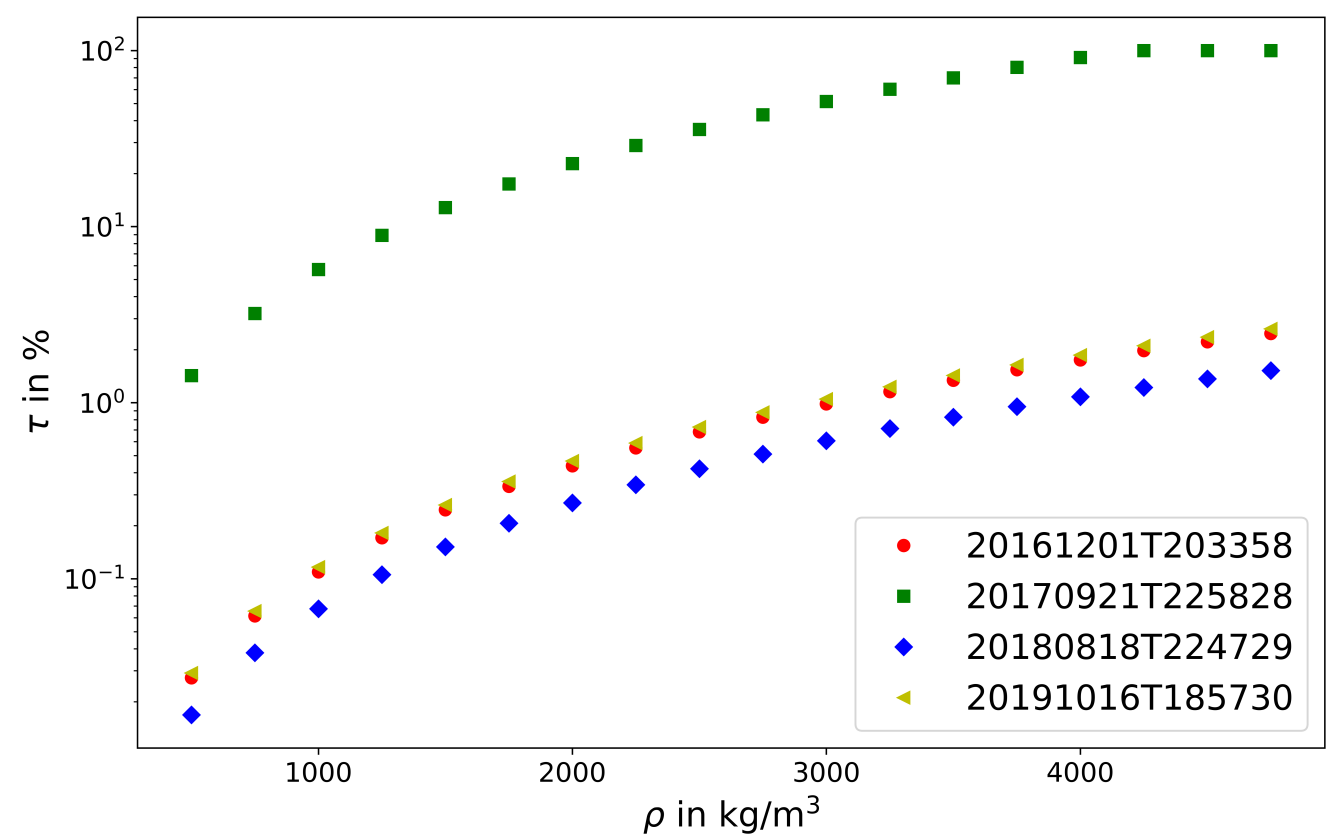

Fig. 2: Luminous efficiency $\tau$ vs. meteoroid bulk density $\rho$ for the four example FRIPON events presented in Fig. 1 and Table 1 . The red dots represent the results calculated with the data of the meteor recorded on 1. December 2016 at 20:33:58 UT, the green boxes show the ones based on the data of the fireball from 21 September 2017 (22:58:28 UT), the blue diamonds correspond to the meteor detected on 18 August 2018 at 22:47:29 UT, and the yellow triangles to the fireball from 16 October 2019 at 18:57:30 UT.

showers originate from established comets, the densities of the corresponding meteoroids are expected to be quite low, due to the well-studied high porosity of comets. Hence, the bulk densities of the associated meteoroids can be presumed quite precisely.

Noteworthy contributions regarding the density of stream associated meteoroids have been made by e.g. Babadzhanov \& Kokhirova (2009) who derived values on the basis of doublestation photographic meteor observations. They found values as low as $400 \mathrm{~kg} / \mathrm{m}^{3}$ for the Leonids and the highest value for their investigated meteoroids was $2900 \mathrm{~kg} / \mathrm{m}^{3}$ for the Geminids. Narziev (2019) determined amongst others meteoroid densities based on simultaneous radar and optical observations of meteors. They found values as high as $3600 \mathrm{~kg} / \mathrm{m}^{3}$ for the Geminids and Southern $\delta$-Aquariids and as low as $600 \mathrm{~kg} / \mathrm{m}^{3}$ for the
Leonids and Orionids. Additionally, they suggest $1300 \mathrm{~kg} / \mathrm{m}^{3}$ for the analysed sporadics. Borovička et al. (2007) provided a density value of $300 \mathrm{~kg} / \mathrm{m}^{3}$ for meteoroids associated with the Draconids in their work, based on stereoscopic optical data of seven shower associated meteors.

Table 2 lists some major streams and the corresponding densities we found in literature and used within our computations. Following Sosa \& Fernández (2009), we assumed 400 $\mathrm{kg} / \mathrm{m}^{3}$ for detected meteoroids associated with streams for which we did not find a proper density value in the literature. Sosa \& Fernández (2009) determined, amongst other things, the densities of ten short-periodic comets. They concluded that their results are particularly meaningful from a statistical point of view, i.e. they can be regarded as the average value of a large 
comet group. This is listed as 'Default' in Table 2.

It has to be mentioned that almost all works we could find in literature determining the density of meteoroids use an assumption for the luminous efficiency in some way. This has to be kept in mind as possible error indicating circular reasoning. Nonetheless, we expect these values to be more precise than using either an average value or only separating sporadics and streams utilizing our default value which is based on observations of comets and the only one without $\tau$ assumption, at least to our knowledge.

We applied the $D_{S H}$-criterion as defined by Southworth \& Hawkins (1963) with a threshold of $D_{S H, M i n}=0.1$ (as mentioned e.g. by Jopek (1993)) to all 3871 confirmed FRIPON events. We computed the similarity between the orbits of the recorded meteoroids with the ones of established stream meteoroids from the database of the IAU Meteor Data Center (accessed 08.08.2020). The orbital elements of the investigated FRIPON meteoroids are computed within the FRIPON pipeline (see Jeanne et al. (2019) and Colas et al. (2020) for details) and extracted from the database for this work. With this method 719 could be classified as stream meteoroids and 1201 as sporadics. Hence, the ratio is about 0.6. The distribution is presented in Fig. 3. The other 1951 events could not be analysed mainly due to too few observational data points. It cannot be ruled out that this introduces some unintended bias.

Assuming the densities for the stream associated meteoroids as listed in Table 2 allows us to compute the corresponding masses with more accuracy. To use these masses for the determination of $\tau$ should yield improved results. As done in Drolshagen et al. (2021, accepted), events that produced unphysical results were excluded from further calculations. This applies to all events with $\mu<0.01, \mu>0.73$, or $\tau>0.99$, as well as events with qualitatively bad light curves or fits (more details on this topic can be found in Drolshagen et al. (2021, accepted)). After this initial discrimination from the initial dataset of 3871 FRIPON events only 281 events were considered for this study. This could introduce a bias.

From our subset of 281 events, 99 were classified as shower meteors and 182 as sporadics. Hence about $65 \%$ of these events are sporadics. The distribution of shower meteors is presented in Fig. 4. It is clearly visible that there were a large number of Perseids and Geminids detected, which confirms the assumptions made in Drolshagen et al. (2021, accepted) based on the velocity distribution.

We analysed the mass distribution of the 281 objects studied in this work. The entry masses range from $10^{-6} \mathrm{~kg}$ to $10^{3} \mathrm{~kg}$ and the median value is $M_{e \text {,median }}=1.85 \cdot 10^{-2} \mathrm{~kg}$. For the improved densities for stream and sporadic objects we studied the distributions of the computed luminous efficiencies and of the shape change coefficients. The $\tau$-values range from about $95 \%$ to ca. $2 \cdot 10^{-4} \%$, whereas about $63 \%$ of the values are in the range of $0.1 \%-10 \%$ and about $73 \%$ are in the range of $0.01 \%-10 \%$. The values for $\mu$ are between 0.28 and 0.73 . The median values for $\tau$ and $\mu$ are $\tau_{\text {median }}=0.99 \%$ and $\mu_{\text {median }}$ $=0.61$. Details and the histograms of the calculated parameters can be found in the Appendix C.
Utilizing the individual densities for each event allows us to refine the dependency of the luminous efficiency on the pre-atmospheric meteoroid mass that was found in Drolshagen et al. (2021, accepted). For that purpose, $\tau$ was plotted against $M_{e}$ in log-log space in Fig. 5. Following Drolshagen et al. (2021, accepted) we plotted the mass dependent relations found by Čapek et al. (2019) and the ones found by Subasinghe \& Campbell-Brown (2018) in the $\tau-M_{e}$ plot for comparison.

To quantify the relation between $\tau$ and $M_{e}$, a weighted least square (WLS) fit was used to optimize the parameters of the function $\tau=\phi \cdot M_{e}^{\Omega}$. The fit produced the following parameters:

$\tau=0.733 \cdot M_{e}^{-0.43}$

The fit is applied to the weighted $\tau$ by the relative error of the value. Please note that at first sight the fit seems slightly high for the presented values. This illusion is due to the chosen form of presentation in the log-log space where the same deviation from the curve seems bigger for values lower than the fit (downwards) than for higher ones, even though they might be the same distance. Since the fit tries to minimise the total deviation from the values, the much larger deviations for higher values are more significant. Visually, however, they appear smaller in this space of representation. In other scales the validity of the fit becomes apparent.

Following Drolshagen et al. (2021, accepted) we also investigated the relation of the refined $\tau$ and the pre-atmospheric velocity $v_{e}$. (The corresponding plot can be found in the supplementary material.) To quantify the relation between $\tau$ and $v_{e}$, a least-square fit was used to optimize the parameters of the function $\tau=b \cdot v_{e}^{a}$. The WLS fit delivered the following curve:

$\tau=0.146 \cdot v_{e}^{1.23}$

Compared with the results, we find by using a constant density value of $\rho=2500 \mathrm{~kg} / \mathrm{m}^{3}$ for all objects (given in Eqs. (13) and (14)), the differences are rather small. For the $\tau-M_{e}$ relation, the slope seems to be very similar. The determined data points seem to be shifted slightly diagonally to the lower right. This agrees with the expectations resulting from the results found in Section 3. For a part of our dataset we could find a shower affiliation. We have assigned a density to these objects that is usually lower than the value of $\rho=2500 \mathrm{~kg} / \mathrm{m}^{3}$ that we used before. We also assigned a lower density of $1300 \mathrm{~kg} / \mathrm{m}^{3}$ to sporadics. As explained in Section 3, a decrease in density results in a shift of the data points in the $\tau-M_{e}$ diagram with $\tau$ and $M_{e}$ in opposite directions. The modest differences for the $\tau-v_{e}$ relations are also in agreement with the results found in Section 3, since the changes in $\rho$ are not as large and influence the relation only slightly as established before.

$\tau=0.492 \cdot M_{e}^{-0.46}$

$\tau=0.020 \cdot v_{e}^{1.71}$

As already mentioned, a relatively high portion of shower meteors make up the dataset examined here. Figure 4 shows that there are 33 Geminids and 23 Perseids. They represent about $12 \%$, and $8 \%$ of the sample, respectively, allowing 
us to investigate these stream meteoroids separately. Figure 6 shows the $\tau-M_{e}$ relation for the Geminids and Perseids. Figure C.5 in the appendix their $\tau-v_{e}$ relations. The WLS fits through the data show:

$\tau=0.228 \cdot M_{e}^{-54}$,

\section{for the Geminids and}

$\tau=0.001 \cdot M_{e}^{-1.07}$,

for the Perseids.

Although the results obtained from the two subsets are limited by the comparably smaller dataset, such as a large scattering, the results are nevertheless useful. Especially since the velocities of the meteoroids of a stream are very similar, the influence of the velocity on the luminous efficiency is no longer a significant factor, considering the $\tau-M_{e}$ relation.)

Since this work focuses on the relationship between $\tau$ and the pre-atmospheric meteoroid mass, $M_{e}$, all derived relations are presented in Table 4 for comparison.

We cannot rule out that the pre-entry meteoroid mass itself may have influenced the detections. An object with small pre-atmospheric mass is only detected if it has a large luminous efficiency, whereas objects with larger masses would be detected regardless of the luminous efficiency. This bias towards larger $\tau$-values is correlated with the detection limits of a system and hence visible in all datasets. Therefore, previous literature suffers from the same weakness. Nonetheless, this does not explain the lack of larger objects with equally large $\tau$-values. If this could be due to statistics - smaller objects are much more frequent and objects with great luminous efficiencies are rare is only speculative so far and this trend has to be investigated further.

The results in Fig. 5 are for the improved assumptions for the densities of the meteoroids. As was found in the previous analysis by Drolshagen et al. (2021, accepted), the calculated values of the luminous efficiency $\tau$ can be very high, approaching $100 \%$. These unphysical values are mainly obtained for some of the smallest masses. In the following we will analyse this behaviour in more detail with the aim to identify the limits of the applied method and to find a parameter regime for which the ablation based model is valid and provides reliable results of the luminous efficiency.

An additional aspect should be emphasized again: the fragmentation of meteoroids. It is possible that we see some of the smaller and fainter events only if they fragment significantly. As discussed in Drolshagen et al. (2021, accepted) and shown e.g. by Subasinghe \& Campbell-Brown (2018) or Ceplecha \& ReVelle (2005), fragmentation induces larger $\tau$-values. This could be another possible observational bias.

\section{Observational bias due to missing parts of the trajectory}

Especially for faint or very fast meteors it is possible that the beginning and/or end of the trajectory was not captured by the cameras. The question is: How were the results affected if only part of the light curve is recorded? We explored these effects by simulating partial light curves and applying our method as detailed in Drolshagen et al. (2021, accepted). As a basis for the light curves, the four exemplary events introduced in Section 3 (see Fig. 1) and listed in Table 1 were adapted. All available intensity data of these events were taken as the complete light curve, assumed to be fully recorded. We excluded the leading and/or last parts of the intensity values from these complete curves and applied our method to find the best values for $\tau$ and $\mu$ to these "incomplete" simulated light curves. The curves were reduced to $95 \%, 90 \%, 85 \%, 80 \%, 75 \%, 70 \%, 60 \%$, and 50 $\%$ of the original length. We applied the method to the beginning and/or the end of the data. This way we were able to see the effects of a missing start of the trajectory and of a not recorded end of the trajectory separately as well as simultaneously. 100 $\%$ used data represents the case in which all available data is utilized (complete light curve), hence the values for $\tau$ and $\mu$ are consistent with the ones listed in Table 1.

The results and details can be found in the supplementary material. Surprisingly, we found that the results stay relatively constant. Especially, if parts of the beginning are excluded from the simulated light curve, the variations in $\tau$ are only moderate.

Since we do not expect our observational data to be perfectly complete, missing parts of the trajectory could be a weak indication for a possible observational bias. In conclusion, we do not see large differences in the resulting $\tau$-values. Also, the $\mu$-values do not vary significantly. Our simulations suggest that our fitting procedure based on the least-squares fit to the luminosity values $I(v)$ is quite robust to missing data parts regardless of which section of the light curve is included. Hence, we do not expect a large effect on the overall results.

\section{Final height of objects}

As mentioned before, it is expected that especially objects with low end heights undergo significant deceleration which can be measured with good accuracy with our method. Hence, we expect qualitatively good data for those events, which were shown to also be the objects with larger pre-atmospheric masses. In the course of this work the objects were separated into subsets according to their final heights, $h_{\text {final }}$. We investigated two subsets in more detail, the events with $h_{\text {final }}<70 \mathrm{~km}$ and the ones with $h_{\text {final }}<55 \mathrm{~km}$. Thus, the events with $h_{\text {final }}<55 \mathrm{~km}$ are also included in the subset for $h_{\text {final }}<70 \mathrm{~km}$. Our complete subset includes 281 events, of those, 149 had $h_{\text {final }}<70 \mathrm{~km}$ and $104 h_{\text {final }}$ $<55 \mathrm{~km}$. As before, $\tau$ was plotted against the pre-atmospheric mass $M_{e}$ and a least-square fit was used to optimize the parameters of the function $\tau=\phi \cdot M_{e}^{\Omega}$ to quantify the relation between $\tau$ and $M_{e}$. The results are shown in Fig. 7 and Fig. 8, respectively. The WLS fit gave:

$\tau=0.083 \cdot M_{e}^{-0.79}$

for the subset with $h_{\text {final }}<70 \mathrm{~km}$ and by only taking the objects in the subset with $h_{\text {final }}<55 \mathrm{~km}$ into account we found:

$\tau=0.272 \cdot M_{e}^{-0.53}$.

As expected, events with small masses were not present in the subsets with low end heights. Since the smaller meteoroids 
Table 2: Meteor showers and utilized densities. From: Narziev (2019), ${ }^{1}$ Sosa \& Fernández (2009), ${ }^{2}$ Borovička et al. (2007), and ${ }^{3}$ Babadzhanov \& Kokhirova (2009).

\begin{tabular}{ccc}
\hline Shower name & Abbreviation & density in $\mathrm{kg} / \mathrm{m}^{3}$ \\
\hline Default & DEF & $400^{1}$ \\
Draconids & DRA & $300^{2}$ \\
Leonids & LEO & 600 \\
Orionids & ORI & 500 \\
Perseids & PER & 1000 \\
Northern Taurids & NTA & $1600^{3}$ \\
Quadrantids & QUA & 2100 \\
Alpha Capriconids & CAP & 1400 \\
Kappa Cygnids & KCG & $2200^{3}$ \\
Northern Delta Aquariids & NDA & $2400^{3}$ \\
Geminids & GEM & 3600 \\
Sporadics & SPO & 1300 \\
Southern Delta Aquariids & SDA & 3500 \\
Southern Virginids & SGV & 5000 \\
Southern Taurids & STA & 1800 \\
\hline
\end{tabular}

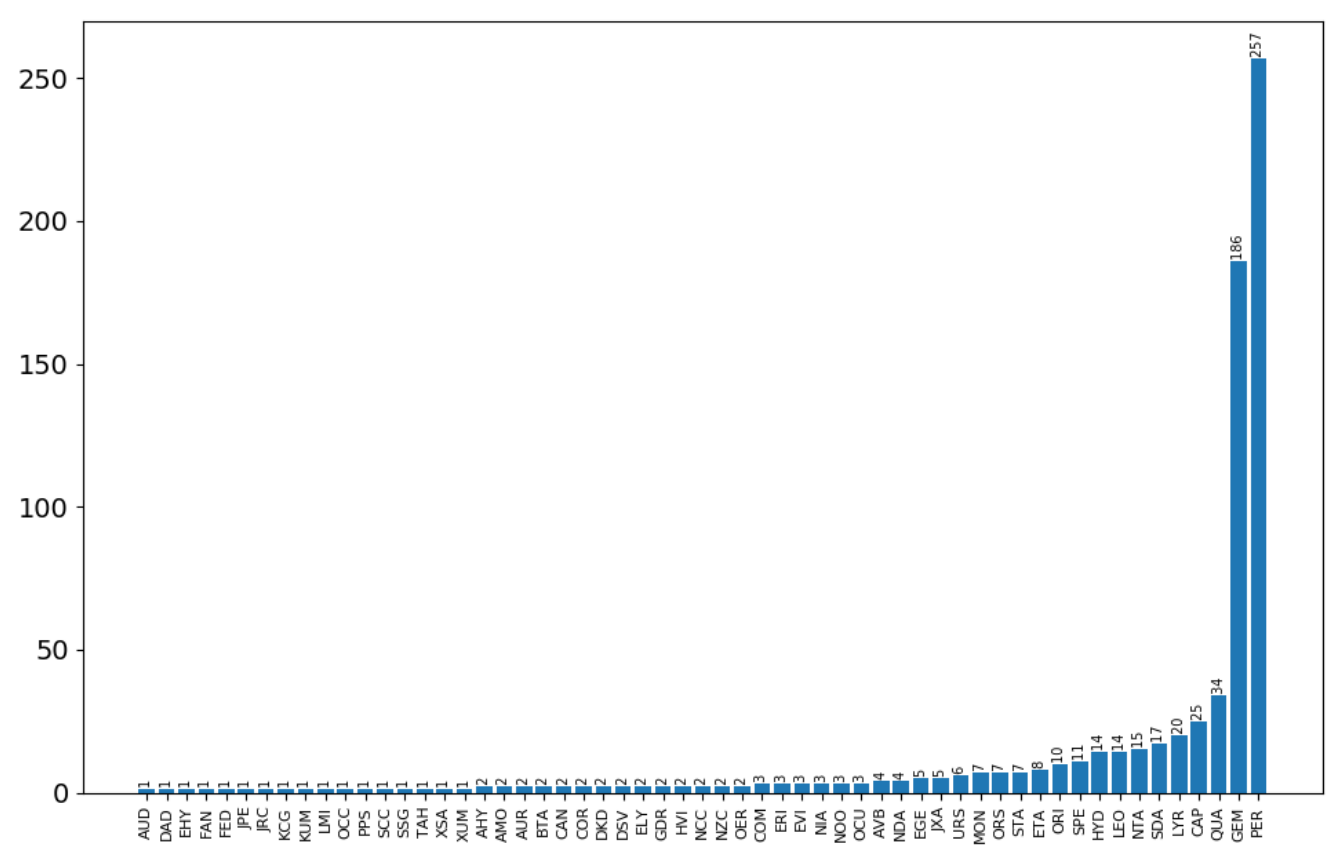

Fig. 3: Distribution of the 719 shower meteors found in the FRIPON dataset of 3871 events.

did show larger luminous efficiencies, these are also absent from these subsets. The fit is not significantly shifted towards lower $\tau$-values. Moreover, the slope found with only the qualitatively good objects that penetrate deep into the atmosphere are even steeper than the one found for the complete dataset. For the subset with $h_{\text {final }}<70 \mathrm{~km}$ the slopes differ even by a factor of two. Hence, for events with low end heights the $\tau$-values would increase even faster with decreasing size. It is clear from the data, that the results still seem to be shifted towards larger luminous efficiencies compared to the ones found by Čapek et al. (2019) and Subasinghe \& Campbell-Brown (2018). The deviation from the results found in these two studies is clearly evident here. We would like to mention that in both those works only 15 , respectively 53, events were investigated. Especially, they also studied much smaller events $\left(10^{-6} \mathrm{~kg}-10^{-4} \mathrm{~kg}\right)$. Hence, the results found in this section may still indicate an observational bias or limited validity of the applied method.

\section{Deceleration}

The applied method is based on the height and velocity information and consequently on the deceleration of the objects. Meteoroids that are decelerated more strongly should be particularly suited for the present method and the data should consequently be of good quality. Hence, the objects' deceleration is an important aspect that we will study in more detail.

To investigate this, the luminous efficiencies are plotted in Fig. 9 over the object's relative velocity change $\left(v_{e}-v_{\text {final }}\right) / v_{e}$ with the corresponding meteoroids' final heights $h_{\text {final }}$ color coded. Our results demonstrate that objects with relatively 


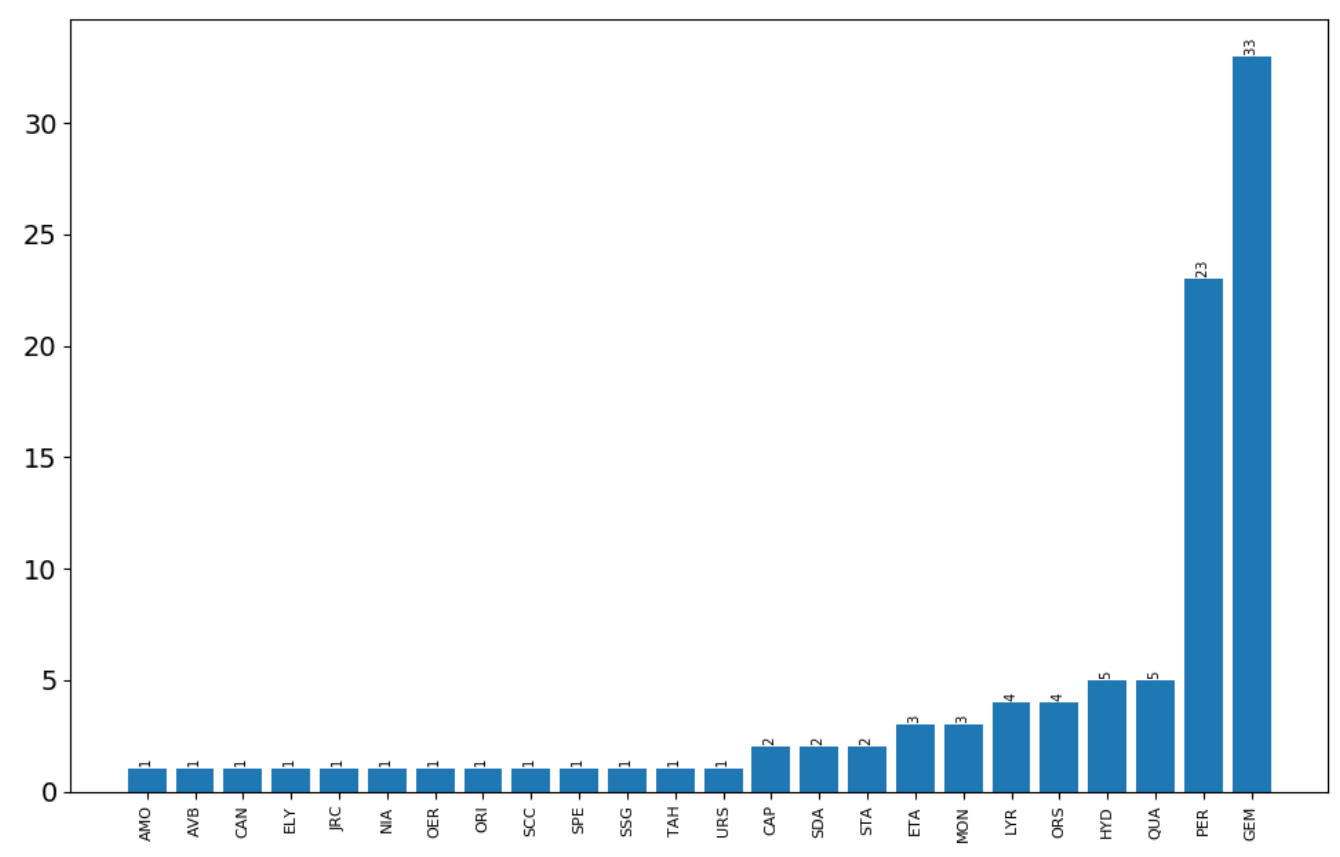

Fig. 4: Distribution of 99 shower meteors in the analyzed subset of 281 meteors.

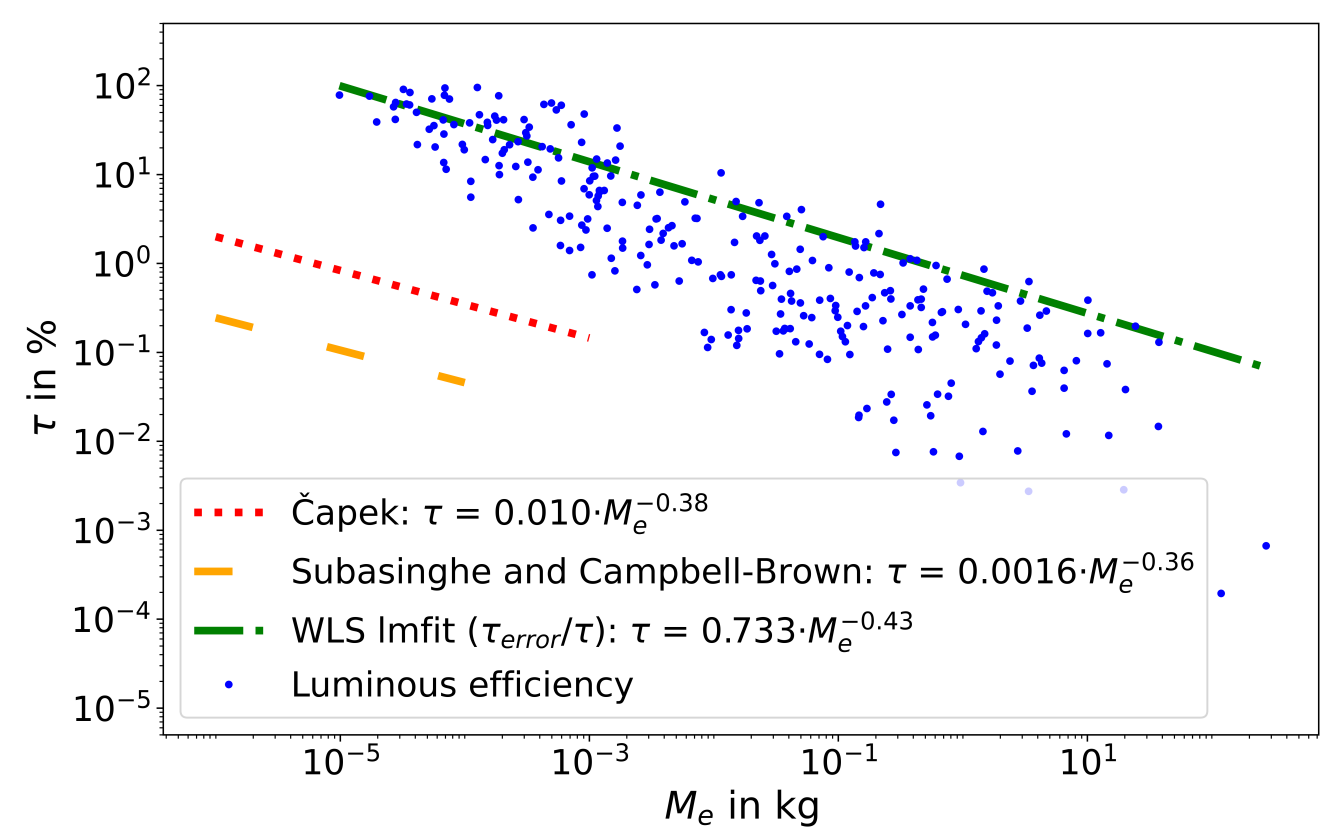

Fig. 5: Luminous efficiencies as derived in this work taking the stream association of the meteoroids into account, plotted over the object's mass with fit through the data displayed in log-log space. Blue dots: values of $\tau$; green dash-dotted line: weighted least squares fit (WLS) applied to the data, the method weights the values by the relative error of the value; red dotted line: the results found by Čapek et al. (2019); orange loosely dashed line: the results found by Subasinghe \& Campbell-Brown (2018).

low measured relative velocity change do not penetrate deep into the atmosphere and disappear at larger final heights. The objects with the largest relative velocity change penetrate deep into the atmosphere. At first glance, it appears that $\tau$ for all relative velocity changes is spread over all represented orders of magnitudes. On closer inspection, however, it is noticeable that the largest and smallest values of $\tau$ only occur for those objects that have a relatively high final height and a rather small relative velocity change. The "hole" in the distribution of data points in Fig. 9 can be explained by the bimodal velocity distribution already shown in Drolshagen et al. (2021, accepted), which is caused by the high proportion of shower meteors and their characteristic velocities and final heights. 


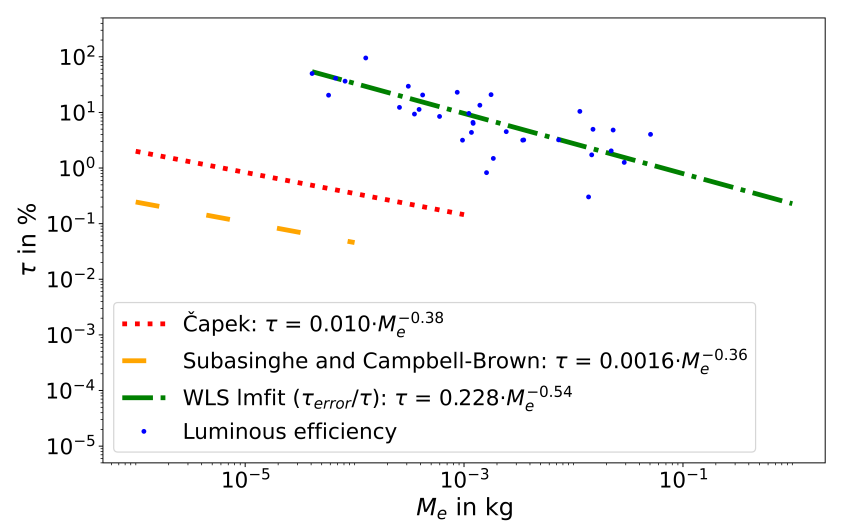

(a) Geminids

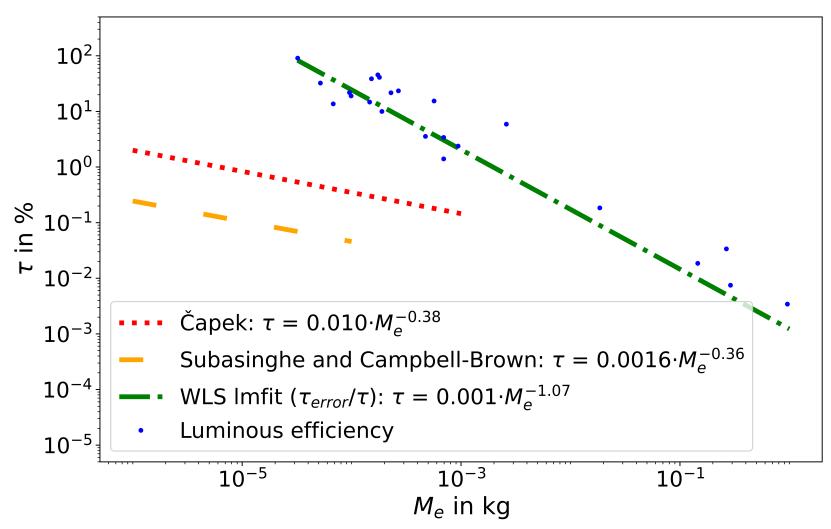

(b) Perseids

Fig. 6: Luminous efficiencies as derived in this work for the Geminids and Perseids in the investigated dataset. The depiction of the data and the meaning of the different curves are as in Fig. 5.

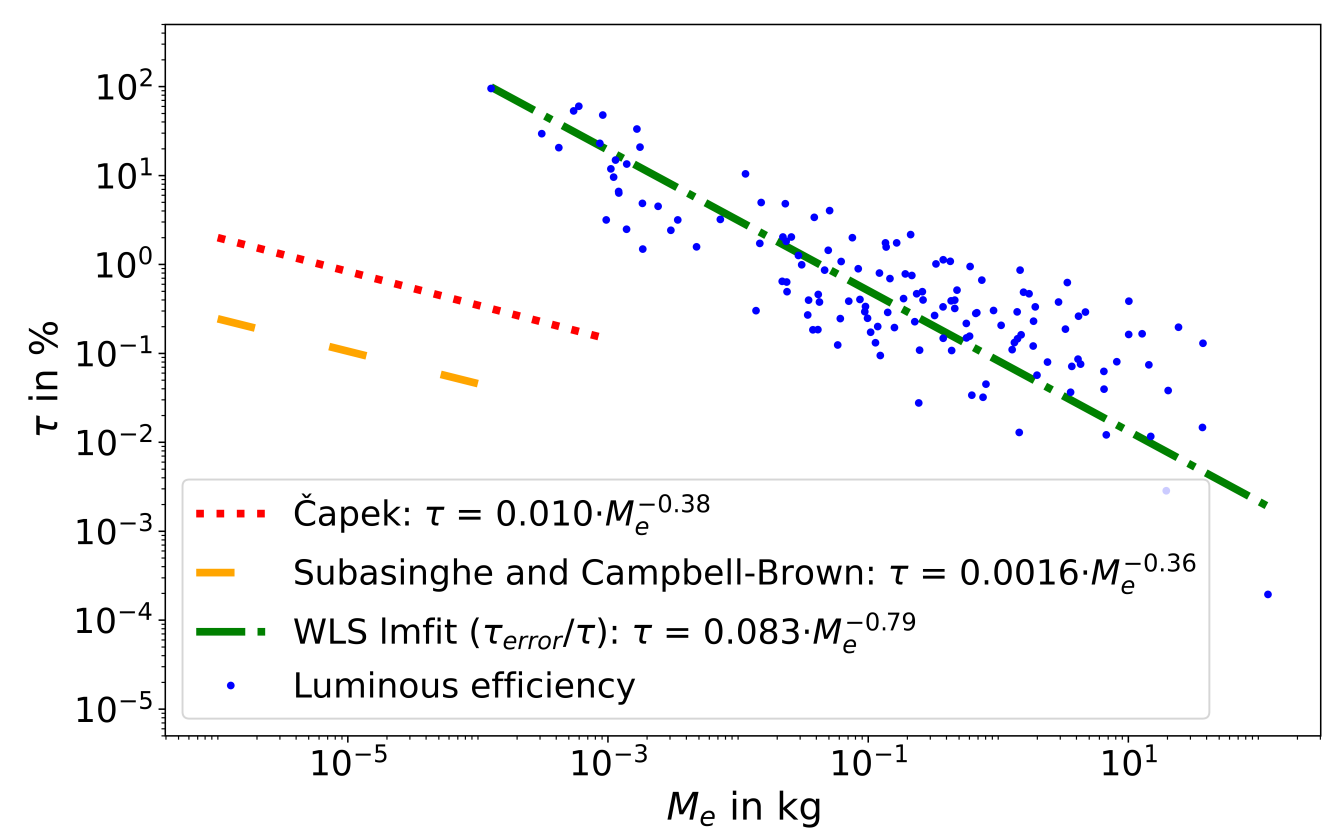

Fig. 7: Luminous efficiencies as derived in this work for the subset of 149 events with $h_{\text {final }}<70 \mathrm{~km}$; The depiction of the data and the meaning of the different curves are as in Fig. 5.

To investigate the influence of the deceleration on the results further, $\tau$ was plotted against the pre-atmospheric mass $M_{e}$ and, as before, a least-square fit was used to optimize the parameters of the function $\tau=\phi \cdot M_{e}^{\Omega}$ to quantify the relation between $\tau$ and $M_{e}$. This is shown in Fig. 10 for only the objects that satisfy the criteria $\left(v_{e}-v_{\text {final }}\right) / v_{e}>80 \%$, which applies to 124 of our objects. The WLS fit produced:

$\tau=2.041 \cdot M_{e}^{-0.35}$

This differs moderately from the values found for all data used (see Eq. (11)), the slope is a lot shallower. Interestingly, the slope is consistent with the ones found by Čapek et al. (2019) and by Subasinghe \& Campbell-Brown (2018) in this case. Still, the results seem to be shifted towards larger luminous efficiencies compared to the ones found by these two studies.

We consider a deceleration as a strong indication of promising events for the present approach. Objects with great deceleration can be well recorded with FRIPON, as these are usually larger objects that penetrate deep into the atmosphere, as can be clearly seen in Fig. 9. As expected, we also found that these objects tend to have larger observation durations. Furthermore, a strong deceleration can be decisive as to how well events can be evaluated with our method.

\section{Flow regimes in the atmosphere}

The findings in the previous sections provide valuable insight into possible biases of the dataset. The results are encouraging but another question on the range of validity of the applied 


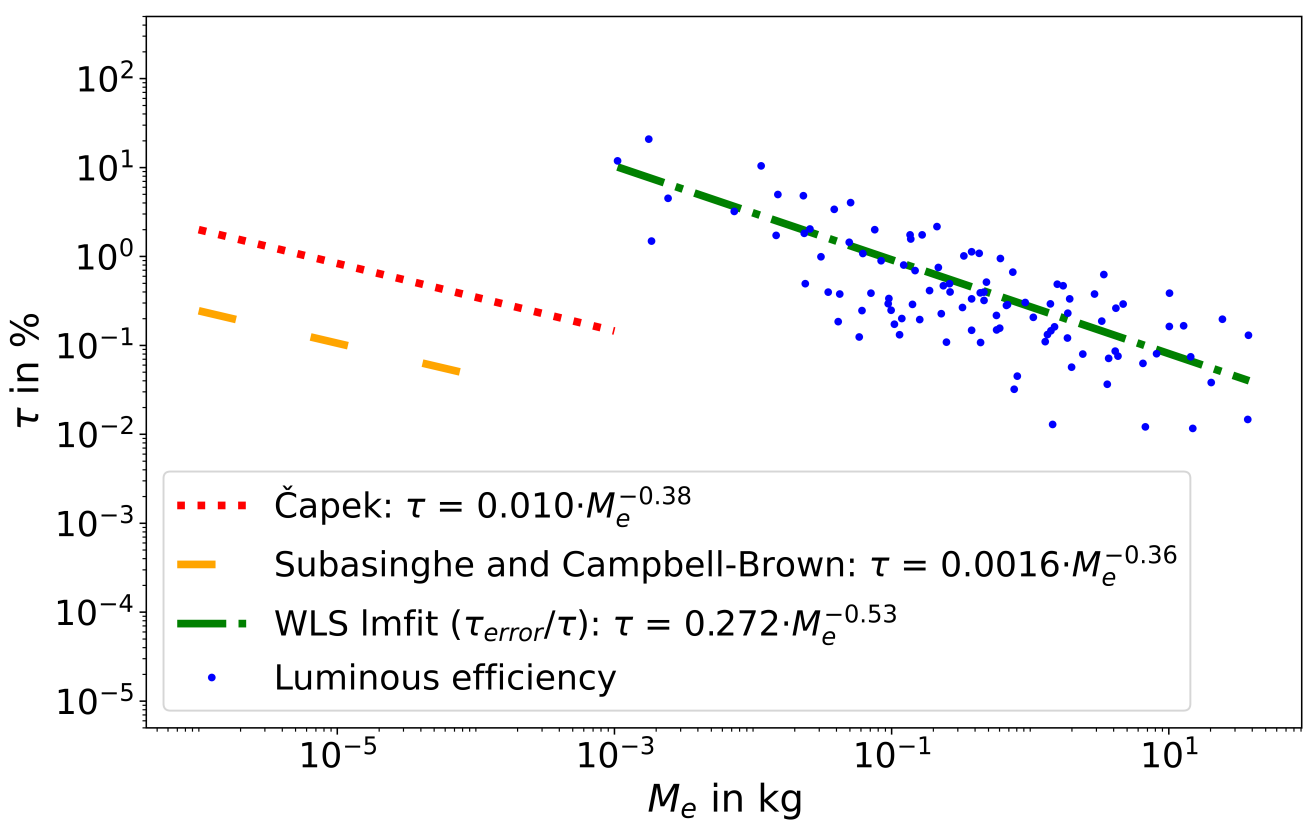

Fig. 8: Luminous efficiencies as derived in this work for the subset of 104 events with $h_{\text {final }}<55 \mathrm{~km}$; The depiction of the data and the meaning of the different curves are as in Fig. 5.

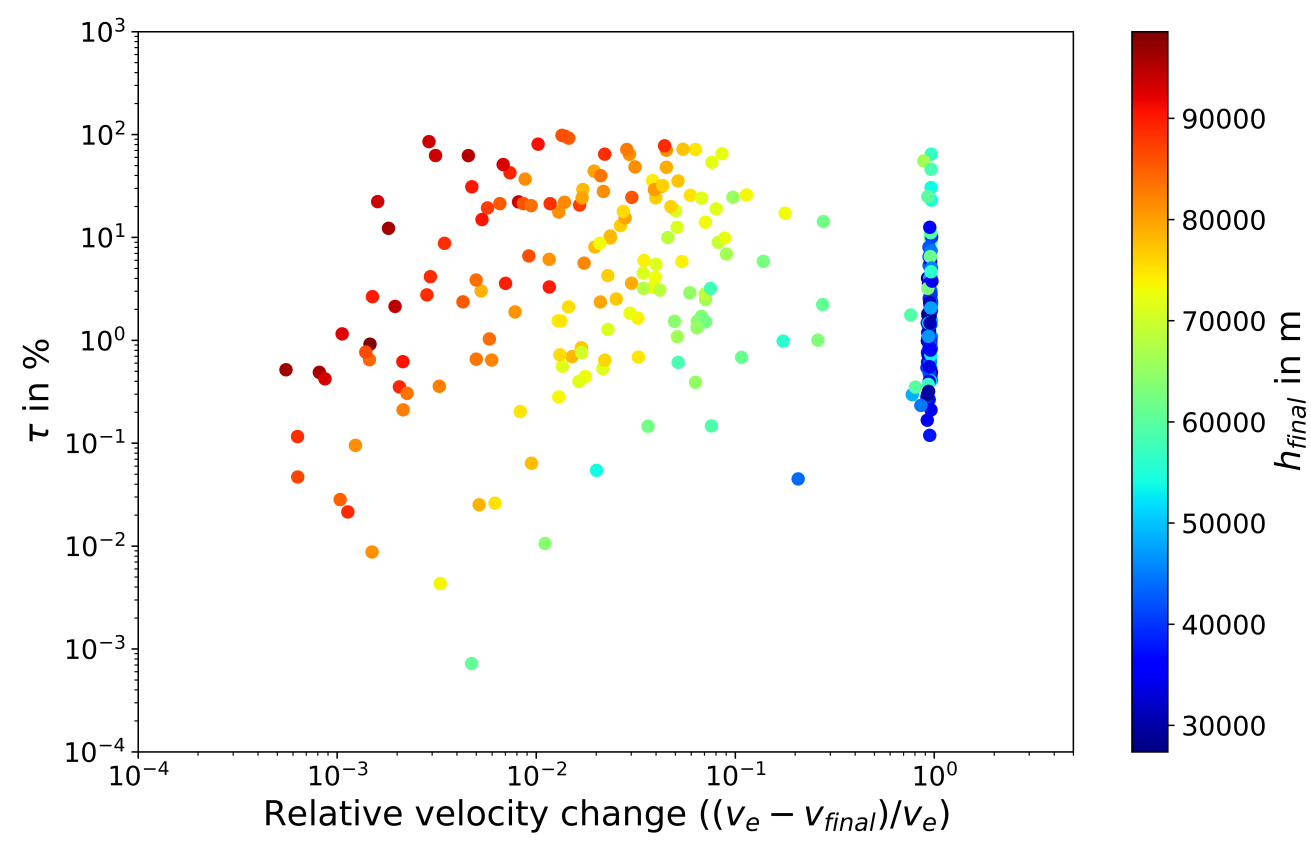

Fig. 9: Luminous efficiencies as derived in this work plotted over the object's relative velocity change for the analyzed objects with the corresponding meteoroids' final heights $h_{\text {final }}$ color-coded.

method comes up. It is expected that the results of events located in the continuum flow regime, where the continuum mechanics are applicable, are the most valid. Hence, the analysis should be done for events observed in the continuum flow regime.

The atmosphere changes with altitude regarding the particle composition, temperature, pressure, density, and many other aspects. Accordingly, the interaction of the meteoroid with the atmosphere also changes leading to variations in the meteor parameters. One effect is the height-dependent density of the atmosphere that influences how numerous and how energetic the air particles are that impinge on the moving meteoroid's body. Additionally, some meteoroid parameters, mainly its velocity and size, affect the interaction of the moving body with the surrounding air molecules. Consequently, there 


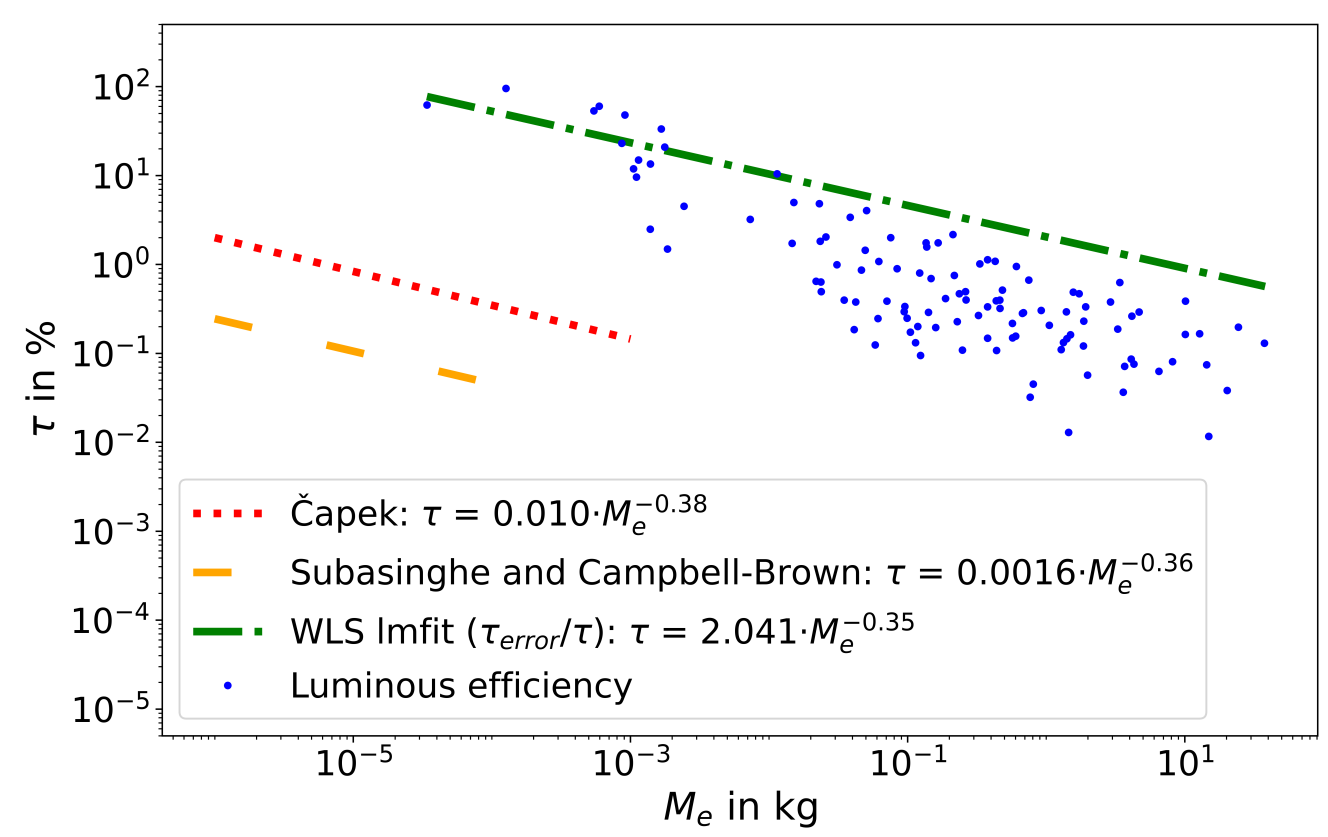

Fig. 10: Luminous efficiencies as derived in this work for the subset of 124 events with $\left(v_{e}-v_{\text {final }}\right) / v_{e}>80 \%$; The depiction of the data and the meaning of the different curves are as in Fig. 5.

are quite a few flight scenarios that are possible. Hence, the entering object may move in different flow regimes during its way through the atmosphere.

A frequently used parameter to characterise the different flow regimes is the Knudsen number $\mathrm{Kn}$. It describes the ratio of the mean free path length $l$ of the molecules (describing the flow conditions around the object) and a characteristic size of the object, in most cases its radius $R$ (Bronshten 1983).

$K n=\frac{l}{R}$

A number of studies have been conducted on the flow regimes of meteoroids. Some noteworthy contributions have been made by e.g. Bronshten (1983), Popova (2000), or Moreno-Ibáñez et al. (2018). In his analysis Bronshten (1983) described how an object that is moving through a medium at hypersonic velocities passes different flow regimes, and then uses this theory to hint at the meteoroid's movement through the Earth's atmosphere. Popova (2000) computed the ablation of non-fragmenting meteoroids, the formation of the vapour cloud around the body, the interaction of the air molecules with this cloud, and studied the flow regimes. They focused on Leonids with a velocity of $72 \mathrm{~km} / \mathrm{s}$ at heights between $90 \mathrm{~km}-110 \mathrm{~km}$ in the size range of $10^{-4} \mathrm{~m}-10^{-1} \mathrm{~m}$. Using their simulations, they reach the conclusion that a dense vapour cloud forms around the body, which prevents direct interaction of air molecules with the meteoroid surface as soon as the height of intensive evaporation is reached. Furthermore, they noticed that the flow regimes shift towards higher altitudes for increasing meteoroid velocities.

Moreno-Ibáñez et al. (2018) investigated data of 24 centimetre-sized objects that were detected simultaneously, optically and by infrasound, based on the previous work of Popova (2000). As described by Popova (2000) MorenoIbáñez et al. (2018) take into account the onset of a vapour cap and a shock wave around the impacting meteoroids. Using real data they further explored the work of Popova (2000).

Since meteoroids enter the Earth's atmosphere at hypersonic speeds, and due to the atmospheric drag produced by its flight through the atmosphere, sound is generated related to atmospheric shock. For impacting objects with diameters larger than about $4 \mathrm{~mm}$, ground measurable shock waves are created. This means that the compressed vapour cloud formed around the object creates a Mach cone and a conical ballistic shock can be assumed (Le Pichon et al. (2009)).

These works are especially interesting since the formation of the vapour cap and the shock wave may play a relevant role in the value of $\tau$.

To analyse meteoroids' flow regimes, Bronshten (1983) defines the mean free path length of the air molecules considering the velocity of the meteoroid. Depending on the movement of the object through the air the mean free path length of the molecules changes due to the impinging and reflected or evaporated air molecules. The used reference frame is the one of the meteoroid. Combining Eqs. (21), (22) (eqs. (6.13) and (6.5) in Bronshten (1983)) with Eq. (23) for the dynamic viscosity from the U.S. standard atmosphere model (1976) (eq. (51) therein, based on Sutherland (1893)) allows us to compute the Knudsen number Kn of the entering objects with Eq. (24).

$$
\begin{aligned}
& K n \approx \frac{5}{R_{e}} \\
& R_{e}=\frac{v \cdot \rho_{a} \cdot R}{\mu_{v}}
\end{aligned}
$$


$\mu_{v}=\frac{\beta_{K n} \cdot T^{3 / 2}}{T+S}$

$K n \approx \frac{5 \cdot \beta_{K n} \cdot T^{3 / 2}}{v \cdot \rho_{a} \cdot R \cdot(T+S)}$

In these equations $R_{e}$ is the Reynolds number, $v$ the meteoroid velocity, $\mu_{v}$ the dynamic viscosity, $\rho_{a}$ the height-dependent density of the atmosphere, $T$ the height-dependent temperature of the atmosphere, $S$ the Sutherland's constant $(S=110.4 \mathrm{~K})$, and $\beta_{K n}=1.458 \cdot 10^{-6} \mathrm{~kg} \cdot \mathrm{s}^{-1} \cdot \mathrm{m}^{-1} \cdot \mathrm{K}^{-1 / 2}$.

It has to be mentioned that Eq. (21) is only true for cases of evaporation which is a valid assumption for meteor entries and that the evaporation temperature is assumed to be $3000 \mathrm{~K}$. For a detailed evaluation see Bronshten (1983).

According to Moreno-Ibáñez et al. (2018) an increase in mass will cause decreasing Knudsen numbers for observed heights. Therefore, the uncertainties within the size computation are expected to be larger.

The literature usually distinguishes between four different flow regimes, based on the Knudsen number or a combination with other parameters. Since the boundaries of the areas can vary depending on the source, we refer here to the work of Moreno-Ibáñez et al. (2018), whose terminology we adopted. At very high heights the atmosphere's density is low and hence there are almost no collisions between the molecules. This free molecular regime is defined for $\mathrm{Kn}>10$. At slightly lower altitudes, in the flow regime between $0.1<\mathrm{Kn}<10$ which is called transitional regime, the number of intermolecular collisions is higher and the molecules' mean free path length is of the same order of magnitude as the object's size. The slip-flow regime is the range around $0.01<\mathrm{Kn}<0.1$. In this layer there is no adhesion of the flow at the boundaries of the surface of the body yet, but the flow velocity has a light tangential component. For even lower altitudes, the flow is regarded as continuous. Thus, the area with $\mathrm{Kn}<0.01$ is called continuum-flow regime (Moreno-Ibáñez et al. 2018).

We expect that the equations on which our method for the calculation of the luminous efficiency is based are best justified in the continuum-flow regime. If applied to other flow regimes it could introduce additional uncertainties or even start to break down. Consequently, results of our computations and the determined $\tau$-values for events with Kn values larger than 0.01 could be less accurate.

Using the U.S. Standard Atmosphere (1976) as a model for the height-dependent density and temperature of the atmosphere (values are taken from http://www.pdas.com/atmos.html on 19. November 2020), we determined the Knudsen numbers of our FRIPON events. We use the height of the first detection, $h_{e}$, the pre-atmospheric velocity and mass. The mass was determined as explained in Section 2 and for the computation of the objects radius a spherical shape was assumed and the density values in accordance to Section 3 used. Moreno-Ibáñez et al. (2018) pointed out that the first confirmed height at which the shock wave was detected is relevant for the determination of the flow regimes but noticed that this is not the earliest altitude at which the shock wave is generated by the meteoroid. Moreover, we point out that we neglect deceleration of our meteoroids in this step. But, since we want to identify those events that generate their meteors in the continuum flow, we still consider it useful to identify those meteors that are in this regime from the beginning, and consequently the whole time during the meteor phenomenon.

We found that objects with low initial heights show low Knudsen numbers. This agrees well with our expectations since Kn depends on the meteoroid's size and the positive dependency of mass and height was already established in the previous sections: larger objects tend to reach lower altitudes. The Knudsen number Kn of our objects plotted against their initial heights, $h_{e}$, can be found in Fig. D.1 in the Appendix. Fig. 11 presents $h_{e}$ against the meteoroid's radius with different symbols for the events indicating in which flow regime the meteoroid is located. None of our events are in the free molecular regime $(\mathrm{Kn}$ $>10)$. Ten are in the transitional regime $(0.1<\mathrm{Kn}<10)$. Most of our objects (178) are in the slip-flow regime $(0.01<\mathrm{Kn}<$ $0.1)$ and 93 lie in the continuum-flow regime $(\mathrm{Kn}<0.01)$. The median value of the Knudsen number for our complete dataset is $\mathrm{Kn}_{\text {median }}=0.017$. The size range of the objects that are located in the continuum flow regime is between about 0.02 $\mathrm{m}$ and $0.3 \mathrm{~m}$

We have also investigated how the results change when we use the height and velocity of the meteoroid at its peak brightness instead of at the entry position to calculate $\mathrm{Kn}$. At peak brightness, the meteoroids have on average already penetrated deeper into the atmosphere and are thus in flow regimes with lower Knudsen numbers. The median in this case would be $\mathrm{Kn}_{\text {median,peak magnitude }}=0.0057$.

If we compare our results with the ones obtained by Moreno-Ibáñez et al. (2018) for their centimetre-sized objects, they found that the first noticeable proof of the existence of the shock wave is at the slip-flow or transitional regime, but they mention that it could have been formed before. They pointed out that this varies when using different scales for the flow regimes or different assumptions for the meteoroids' bulk densities. But they found that the latter did not have much influence on the results.

Since we assume the objects that are located in the continuum-flow regime to be the ones with the most valid results, we extract these events and investigate their $\tau-M_{e}$ relationship, compare Fig. 12. The WLS-fit through the data produces:

$\tau=0.213 \cdot M_{e}^{-0.17}$.

The results are quite similar to the ones found in Section 6. Due to the strong dependency on the Knudsen numbers and the heights of the objects, which can be seen in Fig. D.1, this was to be expected. The events with the smallest masses did not appear in the subsets with $\mathrm{Kn}<0.01$. Since the smaller meteoroids did show larger luminous efficiencies, these events were effectively also excluded from the subset. The fit is not significantly shifted towards lower $\tau$-values. Moreover, the slope found with only the objects with small Knudsen numbers is much shallower than the one found for the complete dataset and even a factor of two shallower than the ones found by Čapek et al. (2019) and by Subasinghe \& Campbell-Brown (2018). Interestingly, the results show luminous efficiencies in similar orders of magnitudes as those 
found by Čapek et al. (2019) and by Subasinghe \& CampbellBrown (2018). Compared to these two studies our luminous efficiency values calculated for this subset of events with $\mathrm{Kn}<0.01$ seem to be shifted towards smaller $\tau$-values.

\section{Discussion}

Deceleration data of meteors observed by FRIPON are used to derive the masses of the corresponding meteoroids. In this study, these results were used to derive the luminous efficiencies by comparison to their brightnesses, in a similar way as demonstrated by Drolshagen et al. (2021, accepted). We investigated various aspects of possible uncertainties and limitations affecting our results and their influences on the luminous efficiency computations in an effort to debias and further improve general mass computations based on luminosities of observed meteors.

The first possible bias considered is the assumed density of the entering object. For the complete dataset and for four exemplary events the dependencies were studied and their influences analysed and discussed. The density was found to be an important parameter, affecting $\tau$ with a power of two. We found that using lower densities yields lower $\tau$-values and obviously larger values for the pre-atmospheric mass $M_{e}$. It is worth noting that the fit of the luminous efficiencies as a function of mass remains quite constant.

To refine the results found in Drolshagen et al. (2021, accepted) shower meteors were identified using the $D$-criterion as introduced by Southworth \& Hawkins (1963). Of all 3871 confirmed events in the FRIPON database 719 could be associated with a known stream and 1201 classified as sporadics. We believe that this significantly improves the computations due to more reliable density values of the individual meteoroids. In the analyzed dataset of 281 meteors, 99 were found to have a shower association. Utilizing the known densities of stream particles for the mass computation, pre-atmospheric masses in the range of $10^{-6} \mathrm{~kg}-10^{2} \mathrm{~kg}$ and luminous efficiencies in the range of $10^{-4} \%-10^{2} \%$ were computed. About $73 \%$ of the $\tau$-values are in the range of $0.01 \%-10 \%$. There was a significant correlation between $\tau$ and the pre-atmospheric velocity $v_{e}$ of the meteor. The dependency could be quantified as $\tau=0.146 \cdot v_{e}^{1.23}$. Furthermore, our results confirm that smaller meteoroids radiate more efficiently. This is evident in the $\tau-M_{e}$ relationship: $\tau=0.733 \cdot M_{e}^{-0.43}$.

Even when using the improved assumptions for the densities of the meteoroids the calculated values of the luminous efficiency $\tau$ can be very high, some up to $100 \%$. These unphysical values are mainly obtained for some of the smallest masses. This behaviour was studied in more detail with the aim to identify the limits of the applied method and to identify a pre-debiased subset for which the ablation based model is valid and provides reliable results of the luminous efficiency.

The possibility of an unintended observational bias due to incomplete trajectory data was investigated since, especially for faint and/or fast meteors, it is probable that either the beginning, end or even both parts of the light curve were not fully recorded. With simulated events the influence on the computations were quantified and discussed. Surprisingly, we found that missing parts do not have a large influence on the derived $\tau$, hence the method used is quite stable with respect to the completeness of the data.

Another aspect studied relates to the limitations of the computation method itself which is based on deceleration data. It is possible that it might not be valid for all meteors. Especially objects with high end heights are less decelerated than those with lower end heights. It was noticed that smaller objects for which we found large luminous efficiencies have high end heights. Using only the events that penetrate deep into the atmosphere excludes particularly small events with large luminous efficiencies. Subsequently, our range of calculated luminous efficiencies decreased. But we also found that the slope of the fit through the data points remained quite similar to previous results for the complete dataset.

In a similar way, we investigated the influence of deceleration on the results. A negative correlation between the relative velocity change and the final height is clearly visible in the presented results. Using only objects that show a relatively large deceleration ensures smaller errors in the recorded data and hence good results, even if the improvement seems negligible. We consider this to be an important aspect indicating qualitatively good data.

Another considered limitation of our approach is based on the inhomogeneity of the atmosphere. Since the conditions around the meteoroid change depending on the atmospheric layer, some equations might not apply for all heights. In the course of this work the flow regime in which an event was observed was investigated based on the mean free path length of the air molecules of the atmosphere which is used to derive the Knudsen number of the entering object. Assuming applicability only in the continuum flow regime, we were able to improve the selection of our dataset. 93 events in our dataset are observed in this regime. Using only those excludes events with high initial heights, small masses, and low deceleration. The largest luminous efficiency values were automatically excluded this way as well.

If we consider all the potentially significant limitations of our data that were discussed, we are left with a subset of 54 events. These have a relative velocity change larger than $80 \%$, a final height lower than $70 \mathrm{~km}$, and a Knudsen number smaller than 0.01 , indicating that these objects move in the continuum flow region. This subset may be considered a pre-debiased set of events. Fig. 13 illustrates the relationship between the three criteria in form of a Venn diagram. The overlapping regions share commonalities while the one lacking an overlap have a distinct criterion. The numbers indicate the number of events that meet exactly these criteria. It can be seen that there is no event that only fulfils the criteria for relative velocity change and flow regime while not meeting the criterion for low final heights. There are ten events that fulfil $\mathrm{Kn}<0.01$ and $h_{\text {final }}<70$ $\mathrm{km}$ but not $\left(v_{e}-v_{\text {final }}\right) / v_{e}>80 \%$. There are also 59 events that fulfil the criteria for relative velocity change and final height but are not in the continuum-flow regime.

The subset of 54 events is made up of events with luminous efficiency values between $0.012 \%$ and $1.1 \%$. If we take only these events and plot their $\tau$ over $M_{e}$ (see Fig. 14) the WLS-fit through the data produces:

$\tau=0.282 \cdot M_{e}^{-0.33}$ 


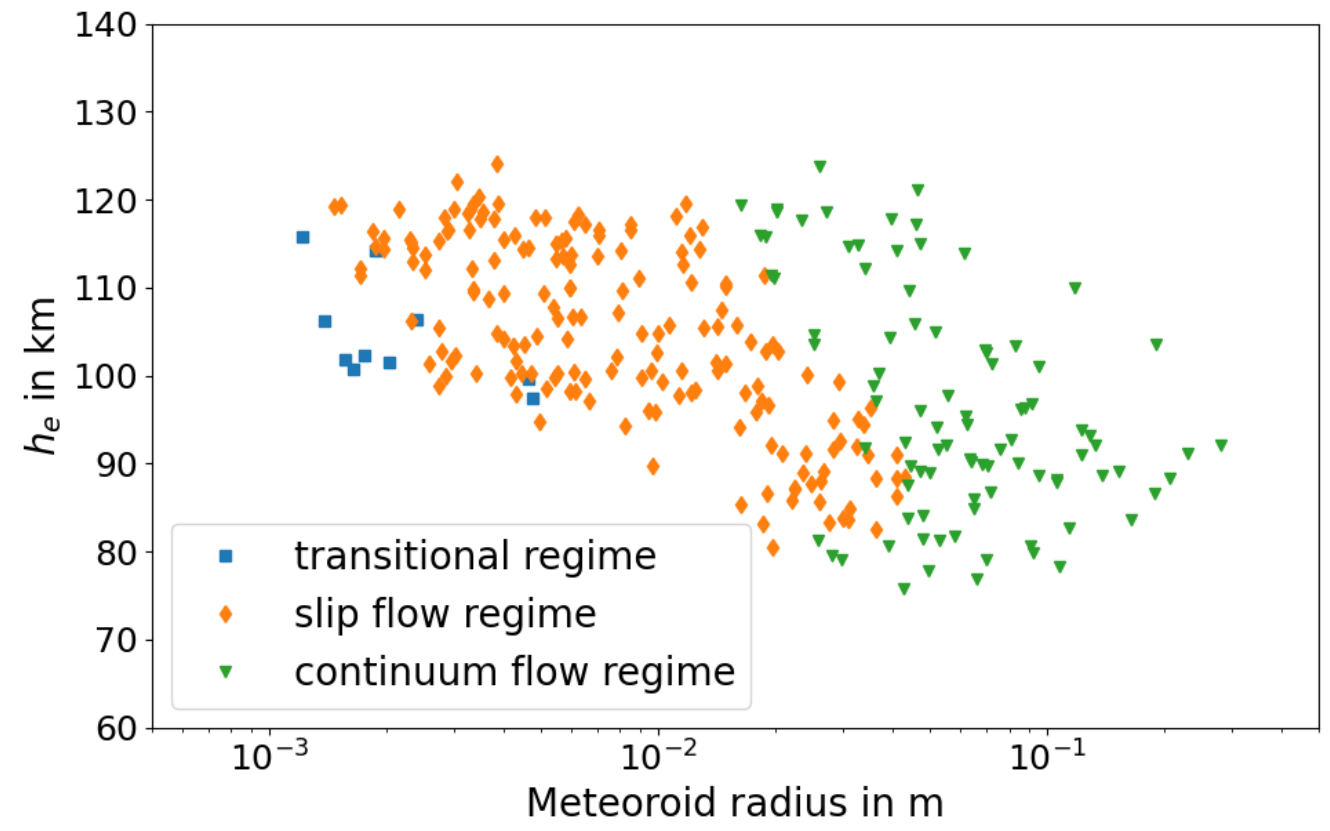

Fig. 11: Initial height $h_{e}$ at which the meteor becomes visible against the meteoroid's radius. The different symbols indicate in which flow regime the corresponding meteoroid is located when the corresponding meteor appears: Blue boxes: transitional regime $(0.1<$ $\mathrm{Kn}<10)$; orange diamonds: slip-flow regime $(0.01<\mathrm{Kn}<0.1)$; green triangles: continuum-flow regime $(\mathrm{Kn}<0.01)$

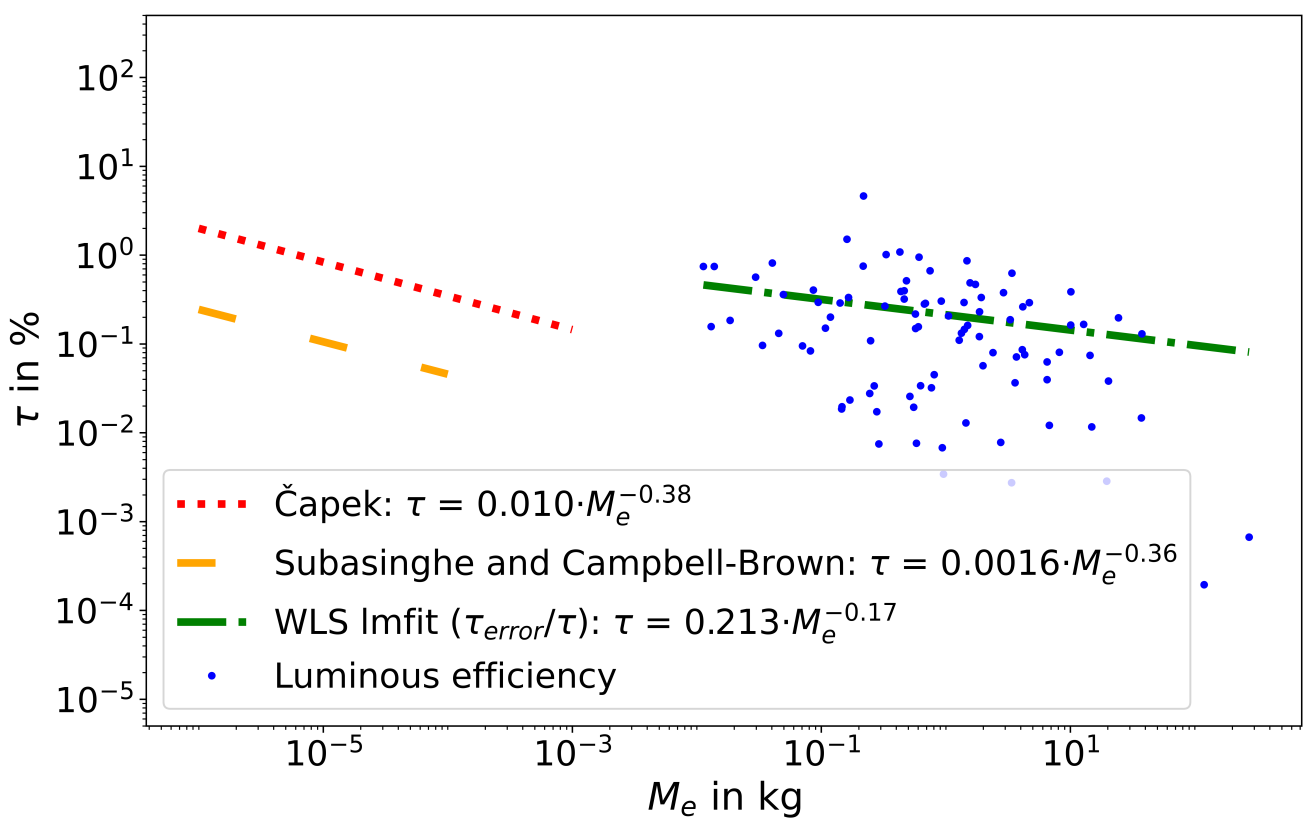

Fig. 12: Luminous efficiencies as derived in this work for the 93 events with $\mathrm{Kn}<0.01$; The depiction of the data and the meaning of the different curves are as in Fig. 5.

Furthermore, the WLS-fit through the data to quantify the $\tau-v_{e}$ relation for this subset was found to be:

$\tau=7.331 \cdot v_{e}^{-1.10}$
The corresponding plot can be found in Fig. E.1 in the Appendix.

The results for the $\tau-M_{e}$ relation are relatively similar to those found in the previous sections. This can be attributed to the fact that most of the remaining events usually either fulfil at least two of the three criteria or fulfil none of the criteria at all. 


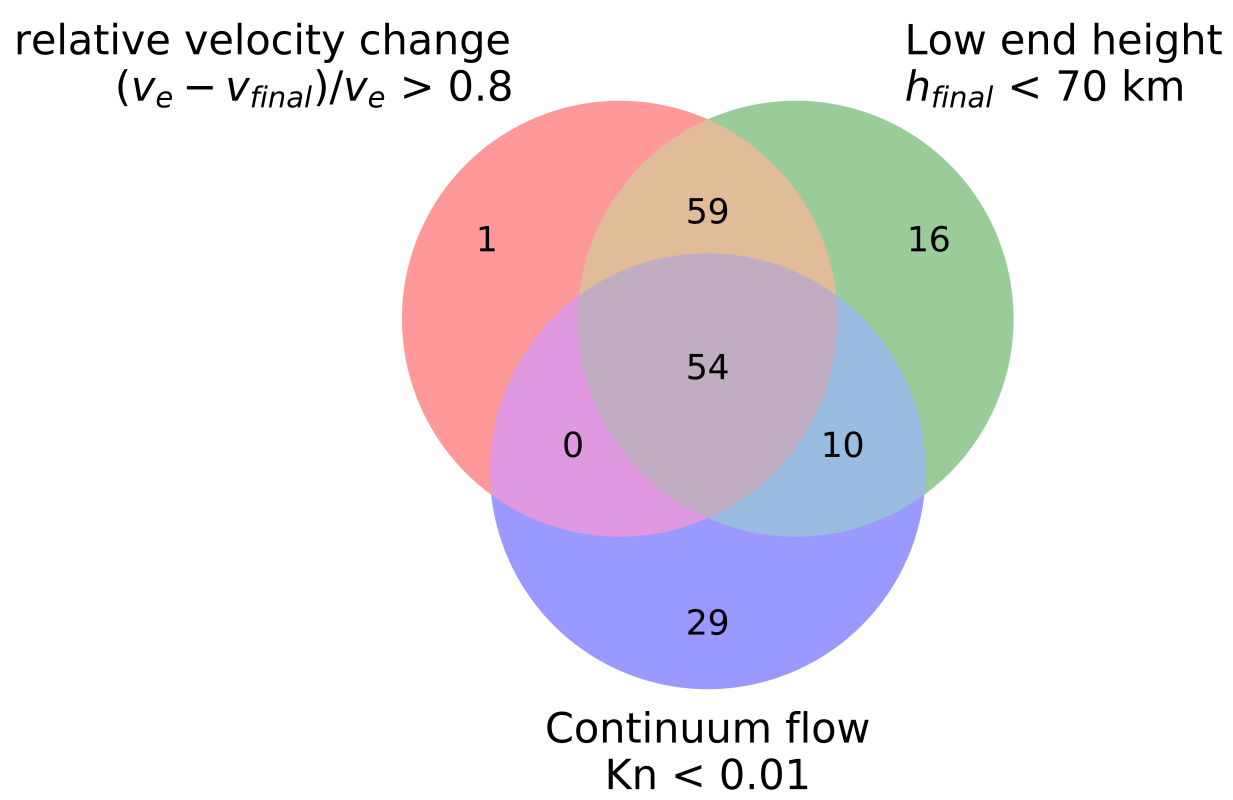

Fig. 13: Venn diagram of the three criteria, with $\left(v_{e}-v_{\text {final }}\right) / v_{e}>80 \%, h_{\text {final }}<70 \mathrm{~km}$, and $\mathrm{Kn}<0.01$, that were used for the pre-debiased dataset of 54 events.

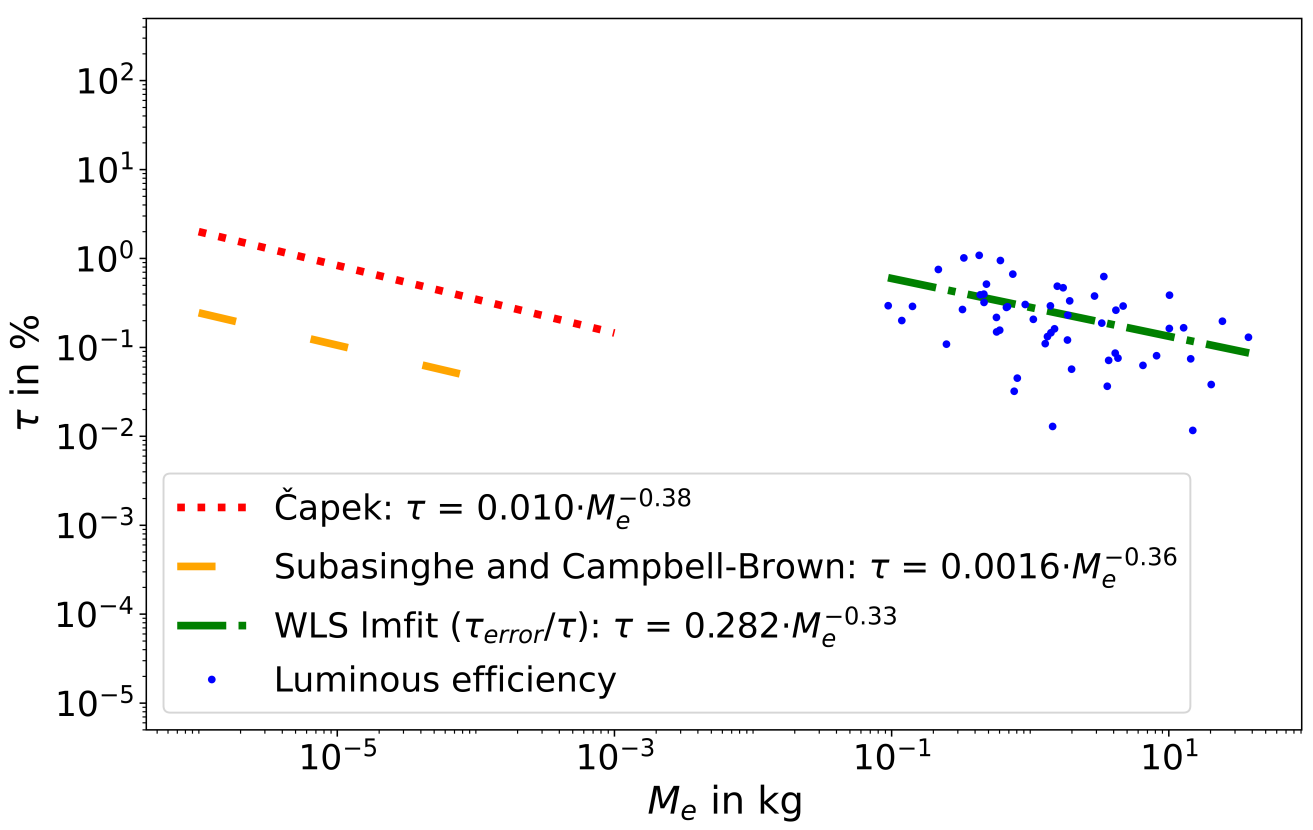

Fig. 14: Luminous efficiencies as derived in this work for the 54 events with $\left(v_{e}-v_{\text {final }}\right) / v_{e}>80 \%, h_{\text {final }}<70 \mathrm{~km}$, and Kn $<0.01$; The depiction of the data and the meaning of the different curves are as in Fig. 5.

Especially the strong correlation between deceleration and final height is worth mentioning again. As discussed in the previous sections, events meeting only one criterion have rather small masses and large $\tau$-values.

For our pre-debiased subset we found $\tau$-values in the range of $10^{-2} \%$ to $10 \%$. Interestingly, the $\tau$-values are now in the same order of magnitude as those found by Čapek et al.
(2019) and by Subasinghe \& Campbell-Brown (2018). The slope obtained is also very similar, even slightly shallower. These findings support the notion that a set-up specific bias for the determination of the luminous efficiency is possible. The smallest objects in each study might only become visible under specific conditions or if they truly have a high luminous efficiency. This could introduce a bias towards higher $\tau$-values for the lower size range considered. We would like to point out, 


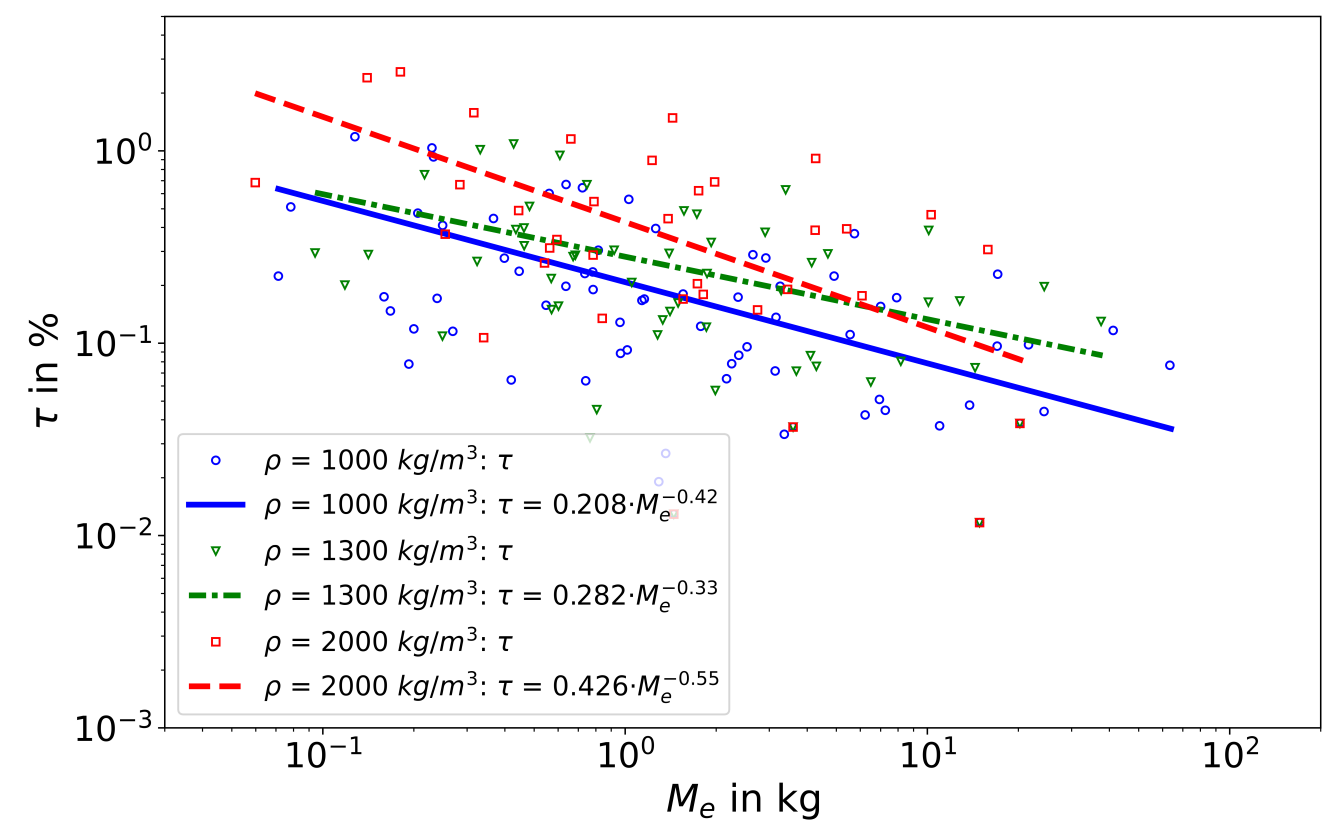

Fig. 15: Luminous efficiencies of 54 events plotted over the objects' pre-atmospheric masses with a fit through the data presented in $\log$-log space. The events were selected according to the following criteria $\left(v_{e}-v_{\text {final }}\right) / v_{e}>80 \%, h_{\text {final }}<70 \mathrm{~km}$, and Kn $<0.01$; and assuming three different sporadic densities; Blue circles: computed values of $\tau$ assuming $\rho_{\text {S poradics }}=1000 \mathrm{~kg} / \mathrm{m}^{3}$ with weighted least squares fit (WLS) applied to the data (solid blue line); Green triangles: (Same as Fig. 14) computed values of $\tau$ assuming $\rho_{\text {S poradics }}=1300 \mathrm{~kg} / \mathrm{m}^{3}$ with weighted least squares fit (WLS) applied to the data (dash-dotted green line); Red boxes: computed values of $\tau$ assuming $\rho_{\text {S poradics }}=2000 \mathrm{~kg} / \mathrm{m}^{3}$ with weighted least squares fit (WLS) applied to the data (dashed red line).

Table 3: Results of the least-square fit on the $\tau$-values in relation to the computed pre-atmospheric velocity, $v_{e}$, and pre-atmospheric mass, $M_{e}$, for varying assumed sporadic densities $\rho_{\text {S poradic }}\left(1000 \mathrm{~kg} / \mathrm{m}^{3}, 1300 \mathrm{~kg} / \mathrm{m}^{3}\right.$, and $\left.2000 \mathrm{~kg} / \mathrm{m}^{3}\right)$, for the pre-debiased dataset containing the 54 events with $\left(v_{e}-v_{\text {final }}\right) / v_{e}>80 \%, h_{\text {final }}<70 \mathrm{~km}$, and $\mathrm{Kn}<0.01$. The results for the weighted least squares method are provided for which the values are weighted by the relative error of the value.

\begin{tabular}{ccc}
\hline$\rho_{\text {S poradic }}$ in $\mathrm{kg} / \mathrm{m}^{3}$ & $\tau-v_{e}$-relation & $\tau-M_{e}$ relation \\
\hline 1000 & $\tau=0.029 \cdot v_{e}^{0.66}$ & $\tau=0.208 \cdot M_{e}^{-0.42}$ \\
1300 & $\tau=7.331 \cdot v_{e}^{-1.10}$ & $\tau=0.282 \cdot M_{e}^{-0.33}$ \\
2000 & $\tau=39.95 \cdot v_{e}^{-1.47}$ & $\tau=0.426 \cdot M_{e}^{-0.55}$ \\
\hline
\end{tabular}

however, that our final dataset contains only 54 events and has therefore been reduced in size compared to the original dataset.

The $\tau-v_{e}$ relation found for this subset differ significantly from the relation found for the complete dataset, see Eq. (12). We even found a negative slope. A possible explanation for this would be the omission of the bimodal shape of the velocity distribution that we have for the entire dataset, but which is no longer seen for the 54 events of this dataset, compare Fig. C.4 and Fig. E.1. For this pre-debiased subset, the velocity lies between: $v_{e, \min }=13.4 \mathrm{~km} / \mathrm{s}$ and $v_{e, \max }=41.1 \mathrm{~km} / \mathrm{s}$. The median is $19.7 \mathrm{~km} / \mathrm{s}$. Of the 54 events in this subset only four were classified as stream meteors. We found one Southern Orionid, one $\alpha$ Virginid, one Southern $\mu$ Sagittariid, and one $\tau$ Herculid. Hence, 50 meteors were classified as sporadics and most identified shower meteors are not included in this subset. These omitted stream meteoroids are those that have high velocities. The higher luminous efficiency of fast events could be explained by the higher energy released. Fast meteoroids generate additional emission lines. These emit more efficiently in certain wavelengths due to the occurrence of the so-called second component of higher temperature (Trigo-Rodríguez et al. 2019). Since we no longer have any fast meteors in this dataset, this second component of higher temperature no longer occurs in the remaining events and they radiate less efficiently. It should also be emphasized that the small number of events in this dataset causes larger inaccuracies in the results.

As shown before the assumed density has a large influence on the results. Hence, we will investigate the effect of density variation on the final dataset as well. More than $90 \%$ of the meteors are sporadics. We assign a standard sporadic density $\left(\rho_{S \text { poradic }}\right)$ to them with a value of $1300 \mathrm{~kg} / \mathrm{m}^{3}$, see Table 2 . We now vary only the sporadic density value for the pre-debiased dataset and observe differing results, as expected. We assumed densities of $1000 \mathrm{~kg} / \mathrm{m}^{3}$ and $2000 \mathrm{~kg} / \mathrm{m}^{3}$. The results for the $\tau-v_{e}$ and the $\tau-M_{e}$ relations are presented in Table 3 and the plot with $\tau$ against $M_{e}$ with applied WLS-fits through the data is shown for all density assumptions in Fig. 15. In this figure, the green triangles are the same as the data points in Fig. 14, as they correspond to $\rho_{S \text { poradic }}=1300 \mathrm{~kg} / \mathrm{m}^{3}$. In line with Section 3 , no obvious pattern in the density varied $\tau-M_{e}$ relations 
Table 4: Results of the least-square fit on the $\tau$-values in relation to the computed pre-atmospheric mass, $M_{e}$, for varying datasets. The results for the weighted least squares method are provided for which the values are weighted by the relative error of the value. If not other mentioned the assumed $\rho$-values are as explained in Section 3

\begin{tabular}{|c|c|c|}
\hline Subset & $\begin{array}{l}\text { Number } \\
\text { of } \\
\text { events }\end{array}$ & $\tau-M_{e}$ relation \\
\hline complete dataset & 281 & $\tau=0.733 \cdot M_{e}^{-0.43}$ \\
\hline complete dataset, $\rho_{\text {All }}=2500 \mathrm{~kg} / \mathrm{m}^{3}$ & 281 & $\tau=0.492 \cdot M_{e}^{-0.46}$ \\
\hline Geminids & 33 & $\tau=0.228 \cdot M_{e}^{-0.54}$ \\
\hline Perseids & 23 & $\tau=0.001 \cdot M_{e}^{-1.07}$ \\
\hline Final height, $h_{\text {final }}<70 \mathrm{~km}$ & 149 & $\tau=0.083 \cdot M_{e}^{-0.79}$ \\
\hline Final height, $h_{\text {final }}<55 \mathrm{~km}$ & 104 & $\tau=0.272 \cdot M_{e}^{-0.53}$ \\
\hline Deceleration, $\left(v_{e}-v_{\text {final }}\right) / v_{e}>80 \%$ & 124 & $\tau=2.041 \cdot M_{e}^{-0.35}$ \\
\hline continuum flow regime, $\mathrm{Kn}<0.01$ & 93 & $\tau=0.213 \cdot M_{e}^{-0.17}$ \\
\hline $\begin{array}{l}\text { pre-debiased dataset, }\left(v_{e}-v_{\text {final }}\right) / v_{e}>80 \%, h_{\text {final }}<70 \mathrm{~km} \text {, and } \\
\mathrm{Kn}<0.01\end{array}$ & 54 & $\tau=0.282 \cdot M_{e}^{-0.33}$ \\
\hline $\begin{array}{l}\text { pre-debiased dataset, }\left(v_{e}-v_{\text {final }}\right) / v_{e}>80 \%, h_{\text {final }}<70 \mathrm{~km} \text {, and } \\
\mathrm{Kn}<0.01 \text {, with } \rho_{S \text { poradic }}=1000 \mathrm{~kg} / \mathrm{m}^{3}\end{array}$ & 54 & $\tau=0.208 \cdot M_{e}^{-0.42}$ \\
\hline $\begin{array}{l}\text { pre-debiased dataset, }\left(v_{e}-v_{\text {final }}\right) / v_{e}>80 \%, h_{\text {final }}<70 \mathrm{~km} \text {, and } \\
\mathrm{Kn}<0.01 \text {, with } \rho_{\text {S poradic }}=2000 \mathrm{~kg} / \mathrm{m}^{3}\end{array}$ & 54 & $\tau=0.426 \cdot M_{e}^{-0.55}$ \\
\hline
\end{tabular}

can be seen (see Table B.1 in the Appendix). The fit assuming $\rho_{\text {S poradic }}=2000 \mathrm{~kg} / \mathrm{m}^{3}$ yields the largest slope. Both are larger than the parameters found for the assumption of $\rho_{\text {S poradic }}=1300$ $\mathrm{kg} / \mathrm{m}^{3}$ (see Eq. (26)). The shallowest slope is seen for the case of the assumption of $\rho_{\text {S poradic }}=1300 \mathrm{~kg} / \mathrm{m}^{3}$. Additionally, in the $\tau-v_{e}$ relations, we see that the value of the slope decreases with increasing $\rho$-value. This result ties well with our previous finding, highlighting that the influence of velocity on luminous efficiency continues to decrease with increasing density.

With slopes of negative values, our findings could indicate that slower meteors radiate more efficiently as long as they are too slow to exhibit the second component of higher temperature. However, given the small size of the dataset and the existing scatter in the data, these results should not be overestimated. Regardless of the validity of these detailed findings, the overall results clearly demonstrate how important the density assumption is on results obtained from meteor research.

This work focuses on the relationship between $\tau$ and the pre-atmospheric meteoroid mass, $M_{e}$. All derived relations for the different investigated subset are presented in Table 4 for comparison.

\section{Conclusions}

For 281 meteors observed by the FRIPON (Fireball Recovery and InterPlanetary Observation Network) the luminous efficiencies were determined. Various limitations that could influence the luminous efficiency computation and inflict uncertainties were investigated. For 99 of our objects we were able to obtain a stream association. By using these stream affiliations of our meteoroids, we refined the calculation of $\tau$ by adopting the known densities of stream meteoroids. We analysed the effects of different assumed densities for sporadic meteoroids and the possibility of an observational bias due to missing parts of the trajectory, the final heights, deceleration, and flow regimes.
The broad implication of our results is the creation of a pre-debiased subset. We expect that the method used is only valid for events that are located in the continuum-flow regime. For these we can be confident that all underlying formulas and most assumptions are valid. These objects typically have both a low final height and a sufficiently high deceleration. FRIPON is able to observe them particularly well since a large velocity change leads to decreasing errors of the recorded data and a low end height allow for easier and longer lasting observations. Debiasing our dataset, we compile a subset of 54 events with a relative velocity change larger $80 \%$, a final height lower $70 \mathrm{~km}$, and a Knudsen number smaller 0.01, indicating that the object is located in the continuum-flow regime. For this pre-debiased subset we found $\tau$-values in the range between $0.012 \%$ and 1.1 $\%$ and the relations of $\tau$ and pre-atmospheric meteoroid velocity $v_{e}$ and mass $M_{e}$ to be: $\tau=7.33 \cdot v_{e}^{-1.10}$ and $\tau=0.28 \cdot M_{e}^{-0.33}$. The latter is in good agreement with recent studies. The negative slope of the $\tau-v_{e}$ relation can be explained by the velocity distribution of the dataset made up of rather slow meteors. The second component of higher temperature no longer occurs in the remaining events and they radiate less efficiently.

Our results indicate that this debiasing method improves the analysis of luminous efficiencies from decelerated meteors. One conclusion that can be drawn is that the luminous efficiency depends on many different aspects and the exact relationship is not yet fully understood. Consequently, one should always be careful when making assumptions for the derivation of this parameter and it will be important that further studies in this direction are conducted.

\section{List of Variables}

$c_{d}$ - drag coefficient

$\rho_{0}$ - gas density at sea level $\left(1.29 \mathrm{~kg} / \mathrm{m}^{3}\right)$

$c_{h}$ - heat-transfer coefficient

$H$ - effective destruction enthalpy

$h_{0}$ - scale height of the Earth atmosphere $(7160 \mathrm{~m})$

$S_{e}$ - pre-atmospheric cross section area of the meteoroid

$A_{e}$ - pre-atmospheric shape factor of the meteoroid 
$M_{e}$ - pre-atmospheric meteoroid mass

$\gamma$ - angle between horizon and trajectory

$v_{e}$ - pre-atmospheric meteoroid velocity

$v_{\text {final }}$ - final meteoroid velocity

$h_{\text {final }}$ - final height of the meteoroid

$h_{e}$ - beginning height of the meteor

$\mu$ - shape change coefficient.

$\tau$ - luminous efficiency

$\rho$ - meteoroid bulk density

$I$ - time dependent light intensity

mag - time dependent absolute magnitude

$v$ - time dependent velocity

$v^{*}$ - dimensionless velocity $v^{*}=v / v_{e}$

$m^{*}$ - dimensionless mass $m^{*}=M / M_{e}$

$\alpha$ - ballistic coefficient

$\beta$ - mass loss parameter

Kn - Knudsen number

$l$ - mean free path length

$R$ - meteoroid radius

$R_{e}$ - Reynolds number

$\mu_{v}$ - dynamic viscosity

$\rho_{a}$ - height-dependent density of the atmosphere

$T$ - height-dependent temperature of the atmosphere

$S$ - Sutherland's constant $(110.4 \mathrm{~K})$

$\beta_{K n}-$ constant $\left(1.458 \cdot 10^{-6} \mathrm{~kg} \cdot \mathrm{s}^{-1} \cdot \mathrm{m}^{-1} \cdot \mathrm{K}^{-1 / 2}\right)$

$\overline{E i}(x)$ - exponential integral $\overline{E i}(x)=\int_{\infty}^{x} \frac{e^{z}}{z} d z$

$y^{*}$ - dimensionless height $y^{*}=h / h_{0}$

Acknowledgements. We thank the European Space Agency and the University of Oldenburg for funding this project. We would especially like to thank Maria Gritsevich for her support and constructive discussions on this matter.

A special gratitude goes to the FRIPON Network for providing the data used for this study and to the FRIPON team for their support of this project. FRIPON was initiated by funding from ANR (grant N.13-BS05-0009-03), carried by the Paris Observatory, Muséum National d'Histoire Naturelle, Paris-Saclay University and Institut Pythéas (LAM-CEREGE). Vigie-Ciel was part of the 65 Millions d'Observateurs project, carried by the Muséum National d'Histoire Naturelle and funded by the French Investissements d'Avenir program. FRIPON data are hosted and processed at Institut Pythéas SIP (Service Informatique Pythéas), and a mirror is hosted at IMCCE (Institut de Mécanique Céleste et de Calcul des Éphémérides / Paris Observatory) with the help of IDOC8 (Integrated Data and Operation Center), supported by CNRS and CNES.

PRISMA is the Italian Network for Systematic surveillance of Meteors and Atmosphere. It is a collaboration initiated and coordinated by the Italian National Institute for Astrophysics (INAF) that counts members among research institutes, universities, associations and schools. The complete list of PRISMA members is available here: http://www.prisma.inaf.it. PRISMA was funded by 2016/0476 and 2019/0672 Research and Education grants from Fondazione Cassa di Risparmio di Torino and by a 2016 grant from Fondazione Agostino De Mari (Savona). FRIPON-Spain is coordinated from the Institute of Space Sciences (CSIC-IEEC). JMT-R, and EPA acknowledge financial support in research project PGC2018-097374-B-I00 (PI: JMT-R) funded FEDER/MCI-AEI.

\section{References}

Anghel, S., Birlan, M., Nedelcu, D.-A., \& Boaca, I. 2019, Romanian Astron. J., 29,3

Audureau Y., Marmo C., Bouley S., et al. 2014, Proceedings of the International Meteor Conference 2014, ed. Rault J.-L. \& Roggemans P.

Babadzhanov, P. B., \& Kokhirova, G. I. 2009, A \& A, 495

Blum J., Schräpler, R., Davidson, B.J.R., \& Trigo-Rodríguez, J.M. 2006, ApJ 652

Borovička, J., Spurný, P., Koten, P. 2007, Astron. Astrophys. 473

Bronshten 1983, Geophysics and Astrophysics Monographs (D. Reidel Publishing Company)

Capek, D., Koten, P., Borovička, J., et al. 2019, A\&A, 625, A106

Ceplecha, Z., \& McCrosky, R.E. 1976, J. Geophys. Res., 81/35

Ceplecha, Z., \& ReVellle, D. O. 2005, MAPS, 40, 1

Colas, F., Zanda, B., Bouley, S., et al. 2014, Proc. of the International Meteor Conference 2014, ed. J.-L. Rault, \& P. Roggemans
Colas, F., Zanda, B., Bouley, S. et al. 2020, A \& A, 644, A53

Drolshagen, E., Ott, T., Koschny, D. 2021, A \& A, accepted

Drummond, J. D. 1981, Icarus, 45, 545

ECSS, European Cooperation for Space Standardization 2008, Space Engineering, Space Environment, ECSS-E-ST-10-04C

Galligan, D. P. 2001, MNRAS, 327, 2

Gritsevich, M. 2008, Doklady Physics, 53, 2

Gritsevich, M. \& Koschny, D. 2011, Icarus, 212, 2

Halliday, I., Griffin, A.A., \& Blackwell, A.T. 1996, MAPS, 31

Hill, K.A., Rogers, L.A., \& Hawkes, R.L. 2005, A \& A, 444, 615

Jeanne, S., Colas, F., Zanda, B., et al. 2019, A\&A, 627, A78

Jopek, T. J. 1993), Icarus, 106, 603

Jopek, T. J., Rudawska, R. \& Bartczak, P. 2008, Earth Moon Planet, 102

Kikwaya J.-B., Campbell-Brown, M., \& Brown, P. G. 2011, A \& A 530, A113

Koschny, D., Drolshagen, E., Drolshagen, S., et al. 2017, PSS, 143

Le Pichon, A., Blanc, E., Hauchecorne, A., 2009, Infrasound Monitoring for Atmospheric Studies, Springer

Levin, B. Yu. 1956, Physical Theory of Meteors and MeteorMatter in the Solar System, USSR Academy of Sciences, Moscow

Levison, H. F., \& Duncan, M. J. 1994, Icarus, 108, 18

Macke, R. J. 2010, Dissertation (University of Central Florida)

Micheli, M. 2013, Dissertation (Universtity of Hawaii at Manoa)

Micheli, M., Tholen, D. J., Jenniskens, P. 2013, Icarus, 267

Milone, E. F., \& Wilson, W. J. F. 2014, Solar System Astrophysics - Planetary Atmospheres and the Outer Solar System (Springer Science \& Business Media New York)

Moorhead, A. V. 2016, MNRAS, 455, 4

Moorhead, A. V., Blaauw, R. C., Moser, D. E., et al. 2017, MNRAS, 472

Moreno-Ibáñez, M., Silber, E. A., Gritsevich, M., \& Trigo-Rodríguez, J. M. 2018, ApJ, 863:2, 174

Narziev, M. 2019, PSS 173

Pätzold, M., Andert, T., Hahn, M., et al. 2016. Nature, 530

Popova, O. P., Sidneva, S. N., Shuvalov, V. V., Strelkov, A. S. 2000, Earth Moon Planets, 82-83

Rudawska, R., Matlovič, P., Tóth, J., Kornoš, L. 2015, PSS, 118

Sosa, A., \& Fernández, J. A. 2009, MNRAS, 393, 1

Southworth, R. B. \& Hawkins, G. S. 1963, Smithsonian Contributions to Astrophysics, 7, 261

Subasinghe, D., Campbell-Brown, M., \& Stokan, E. 2017, PSS, 143

Subasinghe, D., \& Campbell-Brown, M. 2018, ApJ, 155:88

Sutherland, W. 1893, Philosophical Magazine, 5, 36

Stulov, V.P., Mirskii, V.N., Vislyi, A.I. 1995, Aerodinamika bolidov (Aerodynamics of Bolides) (Nauka, Moscow, Russia)

Trigo-Rodríguez, J. M., Rimola, A., Tanbakouei, S., Cabedo-Soto, V., \& Lee, M. R. 2019, Space Sci. Rev., 215:18

Trigo-Rodríguez, J.M. 2019, Hypersonic Meteoroid Entry Physics, eds. Colonna G., Capitelli M. \& Laricchiuta A.

Trigo-Rodríguez, J.M., \& Llorca, J. 2006, MNRAS, 372

Trigo-Rodríguez, J.M., \& Llorca, J. 2007, MNRAS, 375

Trigo-Rodríguez, J.M., \& Llorca, J. 2007b, Adv. Space Res. 39

Trigo-Rodríguez, J.M. \& Blum, J. 2009, PSS 57

Trigo-Rodríguez, J.M., Llorca, J., Borovicka, J., \& Fabregat, J. 2003, MAPS 38

Trigo-Rodríguez, J.M., Llorca, J., \& Fabregat, J. 2004, MNRAS, 348

U.S. Standard Atmosphere 1976, National Aeronautics and Space Administration (U.S. Government Printing Office)

Valsecchi, G. B., Jopek, T. J., \& Froeschlé, Cl. 1999, MNRAS 304, 4

Verniani, F., 1965, Smithsonian Contributions to Astrophysics, 8

Weryk, R. J., \& Brown, P. G. 2013, PSS, 81

${ }^{1}$ University of Oldenburg, Division for Medical Radiation Physics and Space Environment, Germany

e-mail: Esther.Drolshagen@uni-oldenburg.de; e-mail: Theresa.0tt@uni-oldenburg.de

*These authors contributed equally to this work

2 European Space Agency, ESTEC, Keplerlaan 1, 2201 AZ Noordwijk, The Netherlands

3 Chair of Astronautics, TU Munich, Germany

${ }^{4}$ IMCCE, Observatoire de Paris, PSL Research University, CNRS UMR 8028, Sorbonne Université, France.

5 Institut de Minéralogie, Physique des Matériaux et Cosmochimie (IMPMC), Muséum National d'Histoire Naturelle, CNRS UMR 7590, Sorbonne Université, F-75005 Paris, France.

${ }^{6}$ FRIPON (Fireball Recovery and InterPlanetary Observation) and Vigie-Ciel Team, France. 
7 GEOPS-Géosciences, CNRS, Université Paris-Saclay, 91405, Orsay, France.

8 Service Informatique Pythéas (SIP) CNRS - OSU Institut Pythéas UMS 3470, Marseille, France.

9 Aix Marseille Univ, CNRS, CNES, LAM, Marseille, France.

10 INAF - Osservatorio Astrofisico di Torino - Via Osservatorio 20, 10025 Pino Torinese, TO, Italy.

11 Astronomical Institute of the Romanian Academy, Bucharest, RO040557, Romania.

12 MOROI (Meteorites Orbits Reconstruction by Optical Imaging) Astronomical Institute of the Romanian Academy, Bucharest, Romania.

13 SCAMP (System for Capture of Asteroid and Meteorite Paths), FRIPON, UK.

14 Planétarium Rio Tinto Alcan / Espace pour la vie, Montréal, Québec, Canada.

15 Réseau DOME, (Détection et Observation de Météores / Detection and Observation of Meteors), Canada.

16 SPMN (SPanish Meteor Network), FRIPON, Spain.

17 Institute of Space Sciences (CSIC), Campus UAB, Facultat de Ciències, 08193 Bellaterra, Barcelona, Catalonia, Spain.

18 Institut d'Estudis Espacials de Catalunya (IEEC), 08034 Barcelona, Catalonia, Spain.

19 FRIPON-Belgium.

20 Royal Belgian Institute for Space Aeronomy, Brussels, Belgium.

21 Natural History Museum, Burgring 7, A-1010 Vienna, Austria.

22 FRIPON-Austria.

23 Università degli Studi di Torino, Dipartimento di Fisica, Via Pietro Giuria 1, 10125 Torino, TO, Italy.

24 INAF - Osservatorio di Astrofisica e Scienza dello Spazio Via Piero Gobetti 93/3, 40129 Bologna, BO, Italy.

25 INAF - Istituto di Astrofisica e Planetologia Spaziali Via del Fosso del Cavaliere 100, 00133 Roma, RM, Italy.

26 CNR - Istituto di Fisica Applicata Nello Carrara, Via Madonna del Piano, 1050019 Sesto Fiorentino (FI), Italy.

27 Space sciences, Technologies Astrophysics Research (STAR) Institute, Université de Liège, Liège B-4000, Belgium.

28 Università degli Studi di Firenze - Dipartimento di Scienze della Terra, Via Giorgio La Pira, 4, 50121 Firenze, FI, Italy.

29 Natural History Museum, Cromwell Road, London, UK.

30 Dep. Física Aplicada I, Escuela de Ingeniería de Bilbao, Universidad del País Vasco/Euskal Herriko Unibertsitatea, 48013 Bilbao, Spain.

31 Aula EspaZio Gela, Escuela de Ingeniería de Bilbao, Universidad del País Vasco/Euskal Herriko Unibertsitatea, 48013 Bilbao, Spain.

32 FRIPON-Netherlands, European Space Agency, SCI-SC, Keplerlaan 1, 2201 AZ Noordwijk, Netherlands.

33 Osservatorio Astronomico del Righi, Via Mura delle Chiappe 44R, 16136 Genova, GE, Italy.

34 Departament de Quimica, Universitat Autonoma de Barcelona, 08193 Bellaterra, Catalonia, Spain 


\section{Appendix A: The FRIPON data}

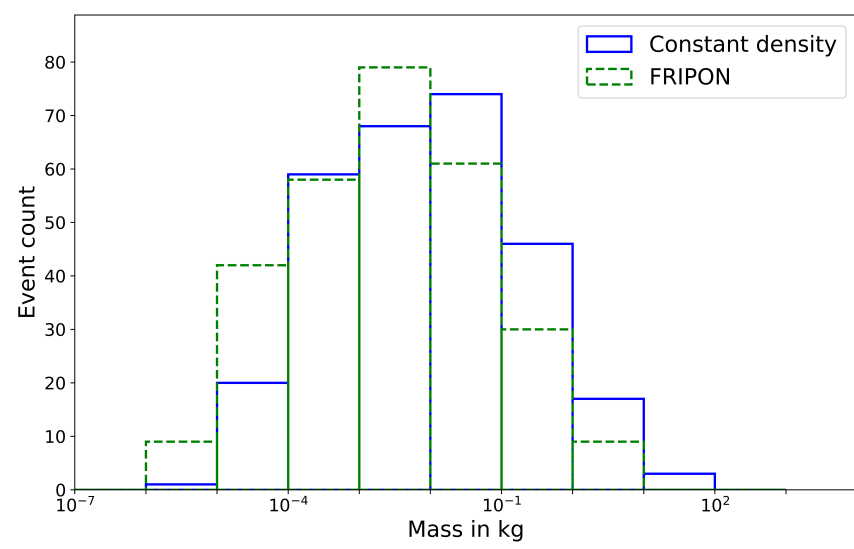

Fig. A.1: The mass distribution of the objects analyzed in this work. The solid blue lined bars show the computed values with an assumption of $\rho=2500 \mathrm{~kg} / \mathrm{m}^{3}$ for all objects. The dashed green lined bars present the masses that were computed by the FRIPON pipeline for the same density estimate.

\section{Appendix B: Densities and mass computation \\ Appendix C: Densities of Stream Meteoroids}

Fig. C.1 shows the pre-atmospheric meteoroid mass distribution of the 281 objects analysed in this work. Compared are the masses computed taking the shower association of the corresponding meteoroids into account as well as the values for $M_{e}$ as a result of an assumed constant standard density $\rho=$ $2500 \mathrm{~kg} / \mathrm{m}^{3}$ for all objects. It can be seen that the largest bin is empty for the distribution with the assumption of $\rho=2500$ $\mathrm{kg} / \mathrm{m}^{3}$. The masses range from $10^{-6} \mathrm{~kg}$ to $10^{3} \mathrm{~kg}$ and from $10^{-6}$ $\mathrm{kg}$ to $10^{2} \mathrm{~kg}$ for a constant density of $\rho=2500 \mathrm{~kg} / \mathrm{m}^{3}$. The median values for the masses based on shower association are $M_{e, \text { median }}=1.85 \cdot 10^{-2} \mathrm{~kg}$ and for a constant density of $\rho=2500$ $\mathrm{kg} / \mathrm{m}^{3} M_{e, \text { median, } \rho_{2500}}=9.20 \cdot 10^{-3} \mathrm{~kg}$. The distribution of the computed luminous efficiencies for the fireballs analyzed in this work is presented in Fig. C.2, the distribution of the shape change coefficients can be seen in Fig. C.3. For comparison, the $\tau$ - and $\mu$-values computed taking the stream association of the corresponding meteoroids into account as well as the values found by using a constant density $\rho=2500 \mathrm{~kg} / \mathrm{m}^{3}$ for all objects are presented. The $\tau$-values range from about $95 \%$ to ca. $2 \cdot 10^{-4} \%$, whereas about $63 \%$ of the values are in the range of $0.1 \%-10$ $\%$ and about $73 \%$ are in the range of $0.01 \%-10 \%$. The values for $\mu$ are between 0.28 and 0.73 . The median values for $\tau$ and $\mu$ are $\tau_{\text {median }}=0.99 \%$ and $\mu_{\text {median }}=0.61$ (for the assumption of $\rho$ $=2500 \mathrm{~kg} / \mathrm{m}^{3}$ they are: $\tau_{\text {median, } \rho_{2500}}=2.09 \%$ and $\mu_{\text {median, }, \rho_{2500}}=$ $0.61)$.

\section{Appendix D: Flow regimes in the atmosphere}

\section{Appendix E: Discussion}

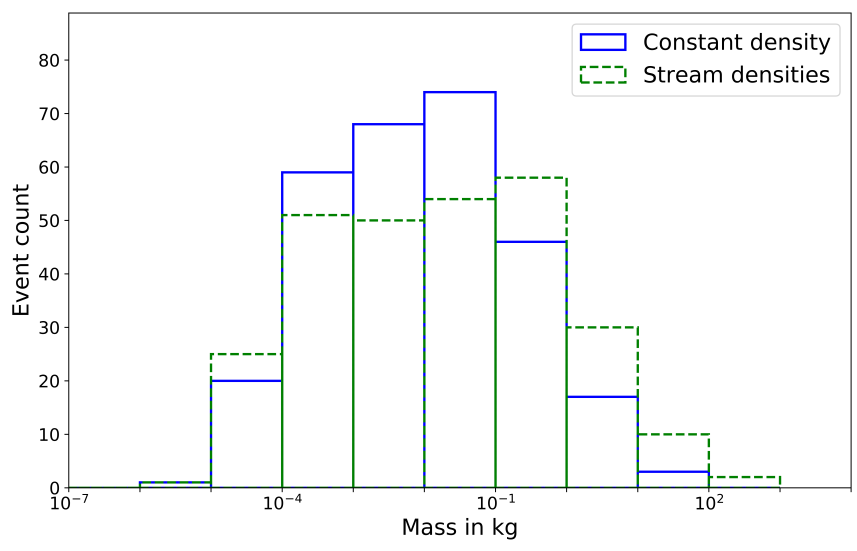

Fig. C.1: The mass distribution of the objects analyzed in this work. The dashed green lined bars present the masses computed taking the stream association of the meteoroids into account. The solid blue lined bars show the values with an assumption of $\rho=$ $2500 \mathrm{~kg} / \mathrm{m}^{3}$ for all objects.

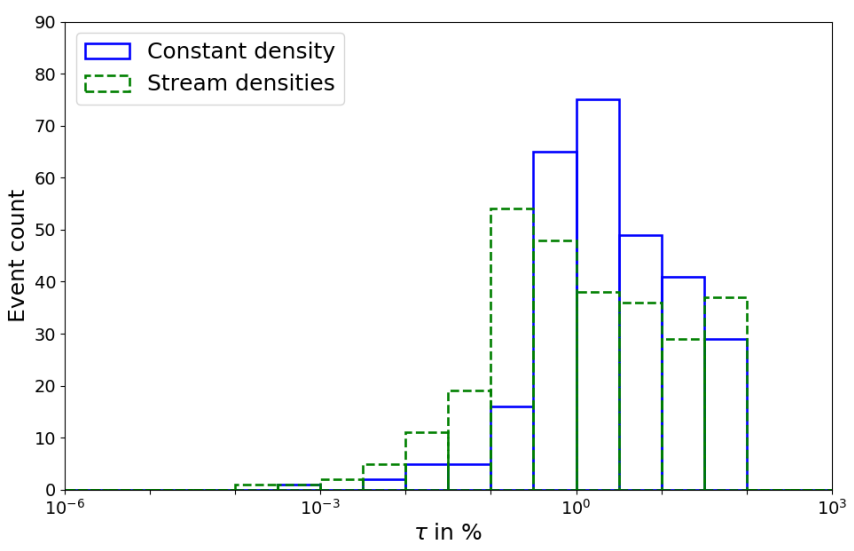

Fig. C.2: The distribution of luminous efficiencies for the fireballs analyzed in this work for two datasets described before. The dashed green lined bars present the $\tau$-values computed taking the stream association of the corresponding meteoroids into account. The solid blue lined bars show the values with an assumption of $\rho=2500 \mathrm{~kg} / \mathrm{m}^{3}$ for all objects. 
Table B.1: Results of the least-square fit on the $\tau$-values in relation to the computed pre-atmospheric velocity $v_{e}$ and pre-atmospheric mass, $M_{e}$, for different assumed meteoroid densities $\rho$. The results for the weighted least squares method are presented for which the values are weighted by the relative error of the value. These results are based on the subset investigated by Drolshagen et al. (2021, accepted) for the density estimation of $\rho=2500 \mathrm{~kg} / \mathrm{m}^{3}$, but six further events were excluded due to the lower and upper boundary for tau. 288 events remain from the 294 evaluated in Drolshagen2021.

\begin{tabular}{ccc}
\hline$\rho$ in $\mathrm{kg} / \mathrm{m}^{3}$ & $\tau-v_{e}$-relation & $\tau-M_{e}$ relation \\
\hline 500 & $\tau=8.443 \cdot 10^{-5} \cdot v_{e}^{3.00}$ & $\tau=0.232 \cdot M_{e}^{-0.54}$ \\
1000 & $\tau=9.449 \cdot 10^{-4} \cdot v_{e}^{2.45}$ & $\tau=0.569 \cdot M_{e}^{-0.44}$ \\
1500 & $\tau=0.019 \cdot v_{e}^{1.77}$ & $\tau=1.049 \cdot M_{e}^{-0.39}$ \\
2000 & $\tau=3.645 \cdot 10^{-3} \cdot v_{e}^{2.18}$ & $\tau=0.723 \cdot M_{e}^{-0.43}$ \\
2500 & $\tau=0.020 \cdot v_{e}^{1.71}$ & $\tau=0.492 \cdot M_{e}^{-0.46}$ \\
3000 & $\tau=0.271 \cdot v_{e}^{1.05}$ & $\tau=0.775 \cdot M_{e}^{-0.41}$ \\
3500 & $\tau=0.205 \cdot v_{e}^{1.17}$ & $\tau=0.856 \cdot M_{e}^{-0.41}$ \\
4000 & $\tau=0.673 \cdot v_{e}^{0.87}$ & $\tau=1.145 \cdot M_{e}^{-0.38}$ \\
4500 & $\tau=1.025 \cdot v_{e}^{0.80}$ & $\tau=1.137 \cdot M_{e}^{-0.39}$ \\
\hline
\end{tabular}

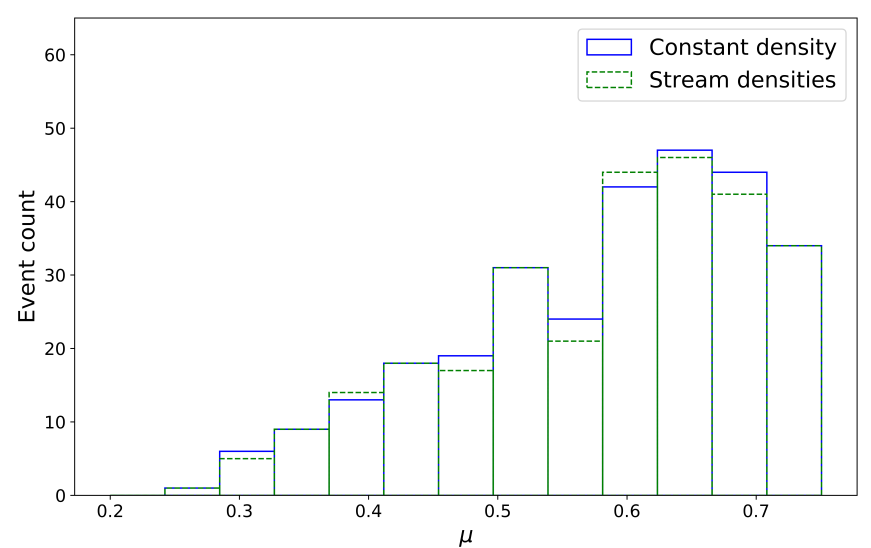

Fig. C.3: The distribution of shape change coefficients for the fireballs of two datasets analyzed in this work. The dashed green lined bars present the $\mu$-values computed taking the stream association of the corresponding meteoroids into account. The solid blue lined bars show the values for $\mu$ with an assumption of $\rho=$ $2500 \mathrm{~kg} / \mathrm{m}^{3}$ for all objects.

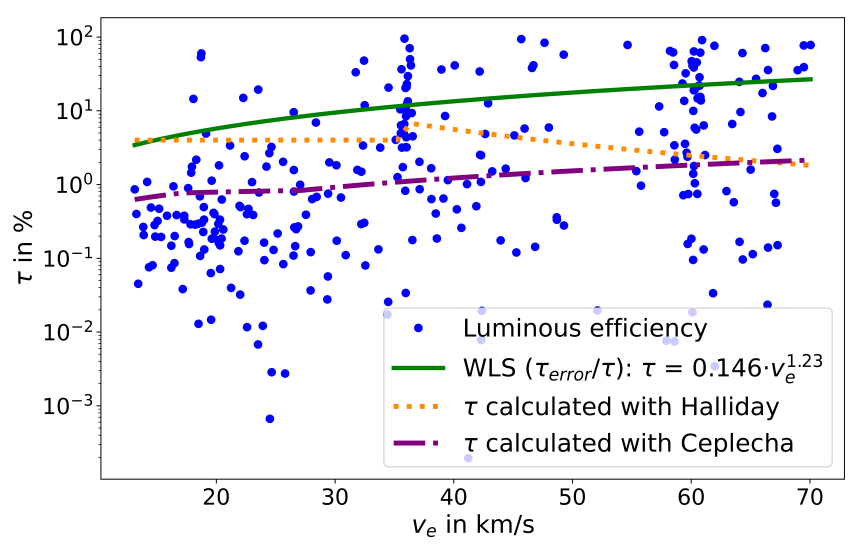

Fig. C.4: Luminous efficiencies as derived in this work taking the stream association of the meteoroids into account, over the initial velocity of the objects with fits through the data displayed in semi-log space. Blue dots: values of $\tau$; green solid line: weighted least squares fit (WLS) applied to the data, the method weights the values by the relative error of the value; orange dotted line: the results found by Halliday et al. (1996); purple dash-dotted line: the results found by Ceplecha \& McCrosky (1976). 


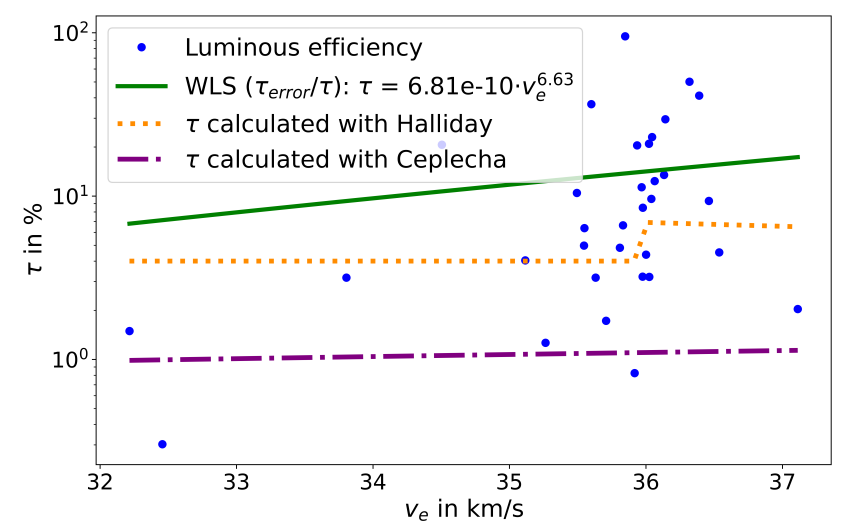

(a) Geminids

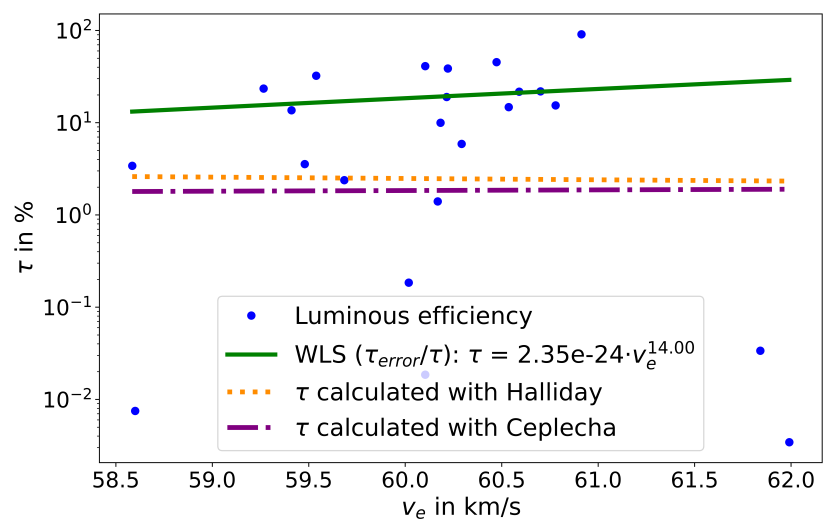

(b) Perseids

Fig. C.5: Luminous efficiencies as derived in this work for the Geminids and Perseids in the investigated dataset, over the initial velocity of the objects with fits through the data displayed in semi-log space. The depiction of the data and the meaning of the different curves are as in Fig. C.4.

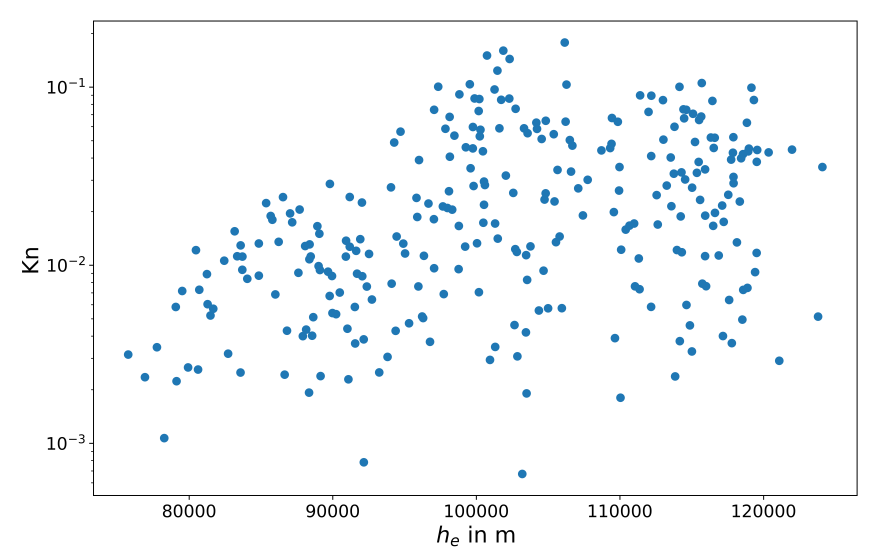

Fig. D.1: Knudsen number Kn of the objects against their beginning heights $h_{e}$.

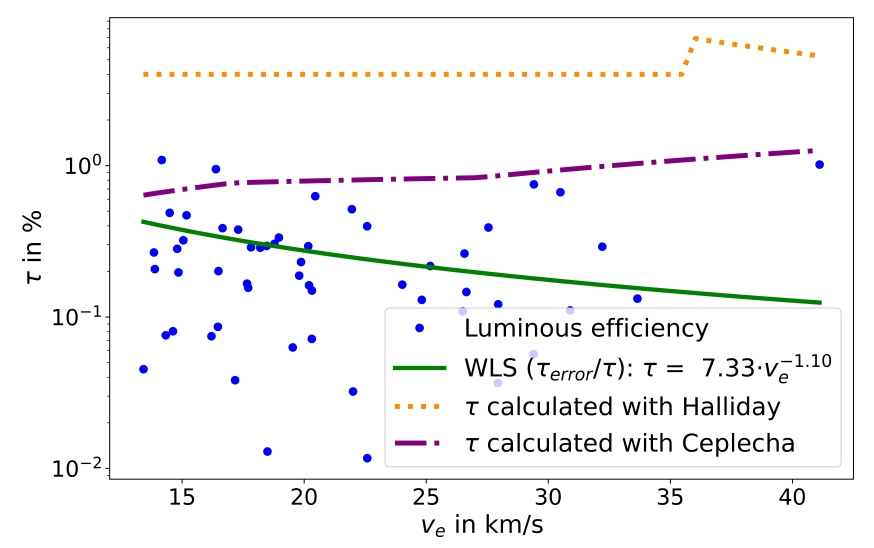

Fig. E.1: Luminous efficiencies as derived in this work over the initial velocity of the 54 events with $\left(v_{e}-v_{\text {final }}\right) / v_{e}>$ $80 \%, h_{\text {final }}<70 \mathrm{~km}$, and $\mathrm{Kn}<0.01$; The depiction of the data and the meaning of the different curves are as in Fig. C.4. 